\title{
REDUCED-ORDER REFERENCE MODELS FOR ADAPTIVE CONTROL OF SPACE STRUCTURES
}

\author{
A Thesis \\ presented to \\ the Faculty of California Polytechnic State University \\ San Luis Obispo
}

\author{
In Partial Fulfillment \\ of the Requirements for the Degree \\ Master of Science in Aerospace Engineering
}

by

Alexander I. Scherling

June 2014 
(C) 2014

Alexander I. Scherling

ALL RIGHTS RESERVED 
TITLE: $\quad$ Reduced-Order Reference Models for Adaptive Control of Space Structures

AUTHOR: $\quad$ Alexander I. Scherling

DATE SUBMITTED: June 2014

COMMITTEE CHAIR: $\quad$ Eric Mehiel, Ph.D.

Associate Professor

Aerospace Engineering Department

COMMitTeE MEMBER: Kira Abercromby, Ph.D.

Associate Professor

Aerospace Engineering Department

COMmitTeE MemBeR: Paul Choboter, Ph.D.

Associate Professor

Mathematics Department

COMmitTeE MEMBER: Jordi Puig-Suari, Ph.D.

Professor

Aerospace Engineering Department 


\begin{abstract}
Reduced-Order Reference Models for Adaptive Control of Space Structures
\end{abstract}

Alexander I. Scherling

In addition to serving as a brief overview of aspects relevant to reducedorder modeling (in particular balanced-state and modal techniques) as applied to structural finite element models, this work produced tools for visualizing the relationship between the modes of a model and the states of its balanced representation.

Specifically, percent error contour and mean percent error plots were developed that provide a designer with frequency response information absent from a typical analysis of a balanced model via its Hankel singular values. The plots were then used to analyze the controllability and observability aspects of finite element models of an illustrative system from a modal perspective - this aided in the identification of computational artifacts in the models and helped predict points at which to halt the truncation of balanced states.

Balanced reduced-order reference models of the illustrative system were implemented as part of a direct adaptive control algorithm to observe the effectiveness of the models. It was learned that the truncation point selected by observing the mean percent error plot produced the most satisfactory results overall - the model closely approximated the dominant modes of the system and eliminated the computational artifacts.

The problem of improving the performance of the system was also considered. The truncated balanced model was recast in modal form so that its damping could be increased, and the settling time decreased by about eighty percent. 


\section{Acknowledgments}

To my professors, friends, and family - I extend my most sincere gratitude. 


\section{Contents}

List of Tables viii

List of Figures $\quad$ ix

1 Introduction 1

1.1 Background and Motivation . . . . . . . . . . . . . 1

1.2 Relevant Literature . . . . . . . . . . . . . . . . . . . . . . 2

1.3 This Work . . . . . . . . . . . . . . . 6

2 State Space Forms of Finite Element Models $\quad 8$

2.1 Finite Element Models . . . . . . . . . . . . . . . . 8

2.2 Second-Order Modal Form . . . . . . . . . . . . . . . . . 11

2.3 Conversion to First-Order Form . . . . . . . . . . . . . . . . . . 14

2.4 First-Order Equivalent Representations . . . . . . . . . . . . . . . 15

2.5 Modalization of Models in First-Order Form . . . . . . . . . . . . 16

3 Model Reduction $\quad 23$

3.1 Modal Truncation . . . . . . . . . . . . . . . . 23

3.2 Controllability and Observability Gramians . . . . . . . . . . . . 24

3.3 Balanced Truncation . . . . . . . . . . . . . . . . 28

3.4 Modes in the Balanced Truncation . . . . . . . . . . . . . . . 32

4 Parametric Studies of Illustrative Systems $\quad 37$

4.1 Control Law . . . . . . . . . . . . . . . . . . . 37

4.2 Modeling an Illustrative System . . . . . . . . . . . . . . . . . 41

4.3 Investigation of Illustrative System . . . . . . . . . . . . . . . . . 42

4.4 Parametric Study . . . . . . . . . . . . . . . . . 50 
5 Conclusion

5.1 Future Work . . . . . . . . . . . . . . . 60

$\begin{array}{ll}\text { Bibliography } & 61\end{array}$ 


\section{List of Tables}

4.1 Performance data for controlled systems, with 15-mode plant . . . 54

4.2 Performance data for controlled systems, with 30-mode plant . . . 57 


\section{List of Figures}

2.1 Diagram of Euler-Bernoulli beam element. . . . . . . . . . . . . . 10

3.1 Log-scale plot of Hankel singular values for an 8-mode model . . . 29

3.2 Percent error contour plot for an 8-mode model . . . . . . . . . . 36

4.1 Control diagram for DMRAC . . . . . . . . . . . . . . . . 40

4.2 Diagram of beryllium mirror segment . . . . . . . . . . . . . . . . 42

4.3 Percent error contour plots of beam models . . . . . . . . . . . . . 43

4.4 Log-scale HSV lots of beam models . . . . . . . . . . . . . . . . . 44

4.5 Bode plots of beam models . . . . . . . . . . . . . . . . . . 45

4.6 Bode plot with gap . . . . . . . . . . . . . . . . . 47

4.7 Percent error contour plot with truncation line . . . . . . . . . . 48

4.8 Plot of mean percent error versus states truncated . . . . . . . . . 50

4.9 Output signals of controlled systems, with 15-mode plant . . . . . 52

4.10 Output error signals of controlled systems, with 15-mode plant . . 53

4.11 Amplitude spectrums of system outputs, with 15-mode plant . . . 55

4.12 Amplitude spectrums of system outputs, with 30-mode plant . . . 56 


\section{Chapter 1}

\section{Introduction}

\subsection{Background and Motivation}

Cameras, telescopes, laser communications links and other vibration-sensitive payloads pose a unique problem in the design of spacecraft. Disturbances due to thermal expansions, spacecraft maneuvers, and other phenomena may interfere with the devices operation. Additionally, due to the extreme expense of launching even the smallest amount of mass into orbit, space structures are designed to minimize weight, and consequently vibrations can be easily excited by any mechanical motion that may take place during normal operation of the spacecraft or its sensors.

Structural vibration is modeled by a partial differential equation, the wave equation, with exact solutions known for certain special cases with simple geometries. Techniques for producing approximate solutions are used in most engineering applications. One of the most popular classes of these are finite element methods, which are advantageous since they can easily model bodies with com- 
plicated geometries. In a finite element model, the structure is discretized into finitely many parts, or elements, that connect a discrete set of points referred to as nodes. The interactions between nodes follow from the physical laws chosen to govern the elements linking them. The global behavior of the model is found by computing the physical interactions between the elements after the application of the relevant boundary and initial conditions, as well as external forces.

The accuracy of a finite element model can be increased by using a finer discretization, and accurate finite element models of structures can contain many thousands of dynamical states. These models can be wasteful however, in the sense that they are often created in a form that requires the storage of much more information than is necessary to capture the behavior of the physical systems they represent. The large size of these models can also make them unattractive for use in some engineering processes, such as controller design. Additionally the possibility exists that the model may include some erroneous dynamical phenomena that do not correspond to reality, in which case removing these dynamics will increase the model's accuracy. It follows that reducing a model to a smaller one that still retains desired system dynamics can be useful in many engineering applications.

\subsection{Relevant Literature}

This project builds off of the 2003 dissertation of Mehiel [16], who applied balanced model reduction and Direct Model Reference Adaptive Control (DMRAC) to the problem of controlling a deployable optical telescope. Mehiel developed a controller design strategy, which included the production of a reduced-order model of the telescope for use as the DMRAC reference model. 
A variety of methods exist for computing reduced-order approximations of finite element models. In particular, since finite element models of structures are second-order systems of linear ordinary differential equations, there are approaches that leave the model in second-order form, and those that convert it to a first-order form. Since model-reduction methods that rely on first-order forms usually do not guarantee preservation of an underlying second-order form, there is interest in methods that do conserve second-order dynamics, as in [22]. It is also important to note that some methods specifically for obtaining reduced-order finite element models of structures are concerned with the response of a structure to low-frequency and static loads, as opposed to vibrations, which will be more of the focus of this work. A comparison of several methods, some of which are for models for use in low-frequency and static responses can be found in [9]. Of the approaches that work well with vibration dynamics, however, the class of modal methods is particularly notable.

Finite element analysis is linked with modal analysis in a mathematical sense; given a finite element model of a structure, one can compute coordinate transformations that place the structure in modal forms, allowing the designer to view the structure's dynamics as a superposition of all of its modes of vibration. This approach can also be reversed to build structural models from experimental modal data, Iwaniec did in [8]. The modal form of a finite element model also allows for a modal truncation method of model reduction.

In standard approaches to modal truncation, first a model is transformed to a modal form, allowing a designer to choose which structural modes to eliminate from the model. The remaining modes can be preserved with zero approximation error individually. Some approaches to modal truncation differ, however, such as approaches involving specialized eigenvalue algorithms that were the focus of 
Kürschner [10] and others. Specifically, the goal of the eigenvalue algorithm in [10] is to compute a modal form that incorporates only the dominant modes of a finite element model, leaving the rest of the modes truncated by virtue of their non-inclusion. Standard modal approaches can be very effective when it is known that the model itself is accurate. Certain modes occur at frequencies well outside of the system's domain of operating frequencies - these modes can be safely removed from the model with minimal approximation error since they did not contribute much to the relevant dynamics of the system. Modal data does not immediately provide insight into the accuracy of a model, however, motivating Iwaniec [8] and others to consider approaches such as balanced model reduction methods.

Balanced model reduction can be traced to a paper by Moore [18], in which principal component analysis was applied to find a generalized representation for first-order systems that would be convenient for model-order reduction - the balanced representation. In this form, the model's controllability and observability gramians are equal and diagonal, with the diagonal entries referred to as Hankel singular values (or HSV's). The system states are eigenvectors of the gramians, allowing a ranking of the states with respect to their controllability/observability based on the magnitudes of their corresponding HSV's, and subsequent elimination of relatively uncontrollable/observable parts of the model.

Algorithms for obtaining a balanced representation from a first-order representation of a system without directly computing its controllability and observability gramians were found by Laub et al. in [12] - work that presented great gains towards making balanced model reduction approaches more feasible from a computation standpoint. Since then, balanced model reduction has solidified itself as a popular model reduction strategy due to its favorable results. Easy 
to compute error bounds for balanced and truncated models, such as those described by Glover in [6] are also partly responsible for the popularity of this method. These error bounds can guide the designer when they are deciding how many balanced states to truncate if they know how much error they are willing to accept in the reduced-order model. One standard approach to halting the truncation of balanced states relies on the designer visually inspecting a log-scale plot of the HSV's. Other approaches have also been put forward, such an approach suggested by Iwaniec in [8] that relies on the approximate rate of change of the sequence formed by the differences of consecutive HSV's.

Many variations of balanced model reduction techniques have been proposed, in particular approximate balancing methods for systems in both first-order forms as in [23] and second-order forms as in [17] and [7]. Approximate balancing is often presented as an option to the designer for easing the computational burden associated with ordinary balancing. Note that the balancing of systems in second-order form is always approximate, however, in the sense that the traditional controllability and observability gramians are only defined for systems in first-order form. Some investigation also appears to have been done towards achieving a hybrid balanced/modal model reduction scheme similar to the approaches utilizing specialized eigenvalue algorithms, with the goal being to produce a computationally-efficient model reduction algorithm while drawing on the benefits of both approaches to reduce approximation error, as in [2].

Another area of interest in balanced model reduction strategies are approaches that modify the balanced truncation to account for expected input and output frequencies, an approach first considered by Enns [3], with others following such Lin and Chiu [14]. In a sense, these approaches are trying to bring to balanced model reduction an important benefit of modal approaches - their ability to 
retain only the modes that will be most relevant across the range of operating frequencies of the model. By applying input and output filters to the model, inputs and outputs at some frequencies can be made more controllable/observable than others to highlight the ranges of operating frequencies; the controllability and observability gramians then take the effect of the filters into account, altering balancing transformation accordingly. Some ongoing work on this approach has been aimed at reducing the approximation error, with recent gains apparently being made by some, such as Muda et al. in [19].

We will briefly comment that although in this work we will implicitly assume that all models we encounter will be continuous-time systems, some methods and techniques involving discrete-time systems are also interesting and relevant. For example, as discussed by Wang and Safonov in [24], truncating states from a discrete-time model in balanced form results in a reduced-order model that is no longer balanced; considering this, they proposed a "one-step-at-a-time" method in which the system is re-balanced after each state is truncated. This example serves as a reminder for using caution when trying to make generalizations between continuous-time and discrete-time systems.

\subsection{This Work}

This project will introduce, summarize and explore a few methods for and aspects of reduced-order modeling, applied to finite element models of space structures. Specifically, modal truncation and balanced truncation methods will be focused on. Methods for computing modal forms of systems are discussed along with their relevance in system modeling and model reduction. The general theory of balanced model reduction is presented, including the properties of 
the controllability and observability gramians, and a derivation of the balancing transformation.

A tool for use in balanced truncation model reduction is developed that aides in visualizing the relationship between system states in a balanced realization and structural modes, namely a contour plot of percent errors associated with each mode as states are truncated from a balanced model. By its construction, the plot inherently provides a designer with some information regarding the frequency responses the various reduced-order models that can be produced using the usual balanced truncation algorithm. This tool is also used to explore the controllability and observability properties associated with various finite element models of an illustrative system.

Additionally, the basic controller architecture and governing equations for the DMRAC algorithm used by Mehiel in [16] are presented with a short discussion of the relevance of adaptive control methods to the reduced-order finite element models considered in this work. Utilizing the DMRAC algorithm, reduced-order models of the illustrative system were applied to the problem of reducing the settling time and vibration present in a plant subjected to a step input command, with modal methods utilized to enact changes in the system performance. 


\section{Chapter 2}

\section{State Space Forms of Finite Element Models}

\subsection{Finite Element Models}

When viscous damping is assumed, a continuous-time finite element model can be presented as a linear system of second-order ordinary differential equations:

$$
M \ddot{\vec{q}}+D \dot{\vec{q}}+K \vec{q}=F \vec{u}
$$

where $M, D, K$ and $F$ are the mass, damping, stiffness, and actuator matrices respectively, $\vec{q}$ is the vector of node displacements, $q_{i}$, and $\vec{u}$ is the command vector of input signals to the actuators. Additionally, sensors can be placed on the structure to measure linear combinations of node displacements and velocities, producing an output signal, $\vec{y}$ :

$$
\begin{array}{r}
M \ddot{\vec{q}}+D \dot{\vec{q}}+K \vec{q}=F \vec{u} \\
\vec{y}=G \vec{q}+H \dot{\vec{q}}
\end{array}
$$


where $G$ and $H$ are the measurement matrices for position and velocity respectively. In structural finite element models without free-floating members or nonconservative forces, the mass and stiffness matrices $M$ and $K$ are symmetric and positive definite. $M$ and $K$ can be formed element-by-element by additively superimposing elemental mass and stiffness matrices $M_{i}$ and $K_{i}$ for each $i$ th element incorporated into the model.

In the following example, we consider the mass and stiffness element matrices for a beam element derived using Euler-Bernoulli beam theory. These elemental matrices can be found in [11], and have the following forms:

$$
\begin{array}{r}
K_{i}=\frac{I_{i} I_{i}}{l_{i}^{3}}\left[\begin{array}{cccc}
12 & 6 l_{i} & -12 & 6 l_{i} \\
6 l_{i} & 4 l_{i}^{2} & -6 l_{i} & 2 l_{i}^{2} \\
-12 & -6 l_{i} & 12 & -6 l_{i} \\
6 l_{i} & 2 l_{i}^{2} & -6 l_{i} & 4 l_{i}^{2}
\end{array}\right] \\
M_{i}=\frac{m_{i}}{420}\left[\begin{array}{cccc}
156 & 22 l_{i} & 54 & -13 l_{i} \\
22 l_{i} & 4 l_{i}^{2} & 13 l_{i} & -3 l_{i}^{2} \\
54 & 13 l_{i} & 156 & -22 l_{i} \\
-13 l_{i} & -3 l_{i}^{2} & -22 l_{i} & 4 l_{i}^{2}
\end{array}\right]
\end{array}
$$

where $E_{i}$ and $I_{i}$ are the Young's modulus of the $i$ th element's material and second moment of the area for the cross section respectively, and $m_{i}$ and $l_{i}$ are the mass and length of the element. The beam elements will be assembled linearly and end-to-end, with consecutive elements sharing the same node at which they are joined. We will take the convention that the $i$ th beam element connects the $i$ th and $(i+1)$ th nodes of a beam model. The corresponding state vector for the element matrices alternates linear and angular displacements so that the forcedisplacement relationship is the following for a joined pair of stiffness elements: 


$$
\left[\begin{array}{c}
f_{i} \\
m_{i} \\
f_{i+1} \\
m_{i+1} \\
f_{i+2} \\
m_{i+2}
\end{array}\right]=\frac{I_{i} I_{i}}{l_{i}^{3}}\left[\begin{array}{cccccc}
12 & 6 l_{i} & -12 & 6 l_{i} & 0 & 0 \\
6 l_{i} & 4 l_{i}^{2} & -6 l_{i} & 2 l_{i}^{2} & 0 & 0 \\
-12 & -6 l_{i} & 24 & 0 & -12 & 6 l_{i} \\
6 l_{i} & 2 l_{i}^{2} & 0 & 8 l_{i}^{2} & -6 l_{i} & 2 l_{i}^{2} \\
0 & 0 & -12 & -6 l_{i} & 12 & -6 l_{i} \\
0 & 0 & 6 l_{i} & 2 l_{i}^{2} & -6 l_{i} & 4 l_{i}^{2}
\end{array}\right]\left[\begin{array}{c}
x_{i} \\
\theta_{i} \\
x_{i+1} \\
\theta_{i+1} \\
x_{i+2} \\
\theta_{i+2}
\end{array}\right]
$$

where $f_{i}$ and $m_{i}$ are the forces and moments at the $i$ th node, and $x_{i}$ and $\theta_{i}$ are corresponding linear and angular displacements at that node, with the same directionality conventions. The $i$ th element and its nodal forces and moments is depicted in figure 2.1 .

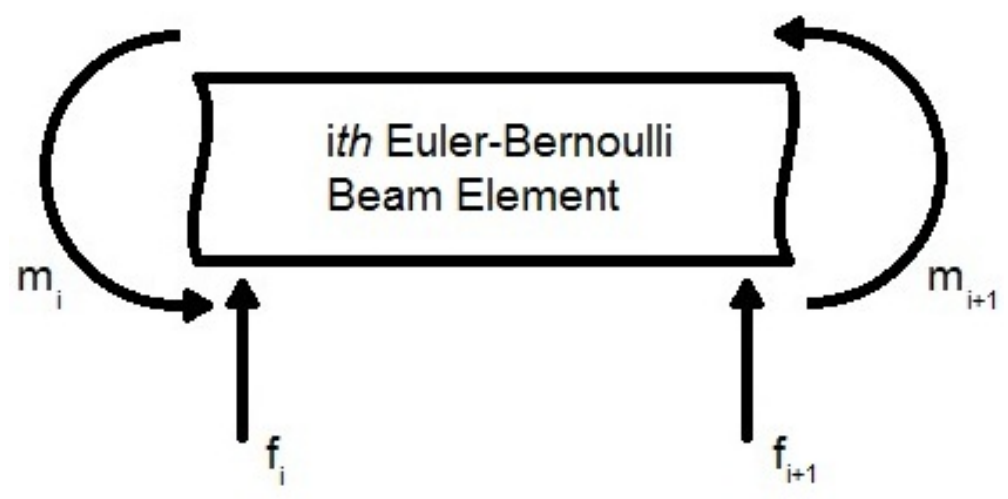

Figure 2.1: Diagram of Euler-Bernoulli beam element. Forces and moments are labeled at the endpoint nodes. The corresponding linear and angular displacements take the same directionality convention as the forces and moments.

The damping matrix $\mathrm{D}$ can be a further approximation that is added in later. This is particularly true for lightly-damped structures, such as those in spacecraft. One method for introducing damping into a finite element model involves transforming the equations in (2.2), with the damping term excluded, 
to an equivalent system of equations - specifically, the usual second-order modal form.

\subsection{Second-Order Modal Form}

We will present a technique for computing the standard modal form of an undamped finite element model in second-order form. Given that the mass and stiffness matrices are symmetric and positive definite, the pair $(M, K)$ can be simultaneously diagonalized by a bilinear coordinate transformation:

$$
\begin{aligned}
& M_{m}=T M T^{\top} \\
& K_{m}=T K T^{\top} .
\end{aligned}
$$

When the coordinate transformation matrix $T$ is selected such that $M_{m}$ is the identity matrix, the representation is said to be modal. The diagonal entries of $K_{m}$ are the squares of the natural frequencies $\omega_{j}$ of the structure, so the structural modes of the system in (2.2) are presented in a decoupled fashion - each mode is represented as a single degree of freedom harmonic oscillator with sensors added:

$$
\begin{array}{r}
\ddot{x}_{j}+\omega_{j}^{2} x_{j}=\vec{\phi}_{j}^{\top} \vec{u} \\
\vec{y}_{j}=\vec{\gamma}_{j} x_{j}+\vec{\eta}_{j} \dot{x}_{j}
\end{array}
$$

where the modal displacement $x_{j}$ describes the net displacement of the $j$ th mode with time, $\vec{\phi}_{j}^{\top}$ is the actuation input vector for the $j$ th mode and $\vec{\gamma}_{j}$ and $\vec{\eta}_{j}$ are the measument vectors for the modal position and velocity repsectively. Note that the subscripts reference indices, but this is not meant to indicate they are summed over. Together $\vec{\gamma}_{j}$ and $\vec{\eta}_{j}$ produce the output signal $\vec{y}_{j}$ for this mode, with the system output $\vec{y}$ formed from the sums of all $\vec{y}_{j}$ (via a sum of two matrix 
products) as depicted in the following $n$-mode system:

$$
\begin{gathered}
{\left[\begin{array}{c}
\ddot{x}_{1} \\
\vdots \\
\ddot{x}_{n}
\end{array}\right]+\left[\begin{array}{ccc}
\omega_{1}^{2} & \ldots & 0 \\
\vdots & \ddots & \vdots \\
0 & \ldots & \omega_{n}^{2}
\end{array}\right]\left[\begin{array}{c}
x_{1} \\
\vdots \\
x_{n}
\end{array}\right]=\left[\begin{array}{c}
\vec{\phi}_{1}^{\top} \\
\vdots \\
\vec{\phi}_{n}^{\top}
\end{array}\right] \vec{u}} \\
\vec{y}=\left[\begin{array}{lll}
\vec{\gamma}_{1} & \ldots & \vec{\gamma}_{n}
\end{array}\right]\left[\begin{array}{c}
x_{1} \\
\vdots \\
x_{n}
\end{array}\right]+\left[\begin{array}{lll}
\vec{\eta}_{1} & \ldots & \vec{\eta}_{n}
\end{array}\right]\left[\begin{array}{c}
\dot{x}_{1} \\
\vdots \\
\dot{x}_{n}
\end{array}\right] .
\end{gathered}
$$

The system in (2.2) is converted to this modal form by the coordinate transformation matrix $T$ (which is sometimes called the modal matrix) as follows:

$$
\begin{array}{r}
T M T^{\top} \ddot{\vec{q}}+T K T^{\top} \vec{q}=T F \vec{u}(t) \\
\vec{y}=G T^{\top} \vec{q}+H T^{\top} \dot{\vec{q}} .
\end{array}
$$

Many variations on the computation of such a coordinate transformation matrix, $T$ exist, but we will use an approach that requires only the ordinary eigenvalue decomposition of a symmetric matrix. This method can be found in [4] and begins with computing the following eigendecomposition:

$$
M^{-1 / 2} K M^{-1 / 2}=V \Omega V^{\top} .
$$

In (2.9) we multiplied $K$ on both sides by $M^{-1 / 2}$ (the symmetric square root of $M^{-1}$ ) for ease of presentation, but in practice one could use two halves of a Cholesky factorization of $M^{-1}$ instead. Each column of the orthogonal matrix $V$ in (2.9) is an eigenvector of the (symmetric) matrix quantity formed on the left-hand side, and $\Omega$ is a diagonal matrix of corresponding eigenvalues. The coordinate transformation matrix $T$ can take the form:

$$
T=V^{\top} M^{-1 / 2}
$$


with the mass and stiffness matrices transforming as follows:

$$
\begin{array}{r}
T M T^{\top}=V^{\top}\left(M^{-1 / 2} M M^{-1 / 2}\right) V=V^{\top} I V=I \\
T K T^{\top}=V^{\top}\left(M^{-1 / 2} K M^{-1 / 2}\right) V=V^{\top}\left(V \Omega V^{\top}\right) V=\Omega .
\end{array}
$$

Note that the eigenvalues on the diagonal of $\Omega$ are the squares of the natural frequencies of the structure.

After the mass and stiffness matrices have been transformed, damping can be easily introduced into the model. Specifically, the harmonic oscillator corresponding to the $j$ th structural mode can be given a damping coefficient, $2 \zeta_{j} \omega_{j}$, so that its damping is proportional to the modal velocity, $\dot{x}_{j}$ :

$$
\begin{array}{r}
\ddot{x}_{j}+2 \zeta_{j} \omega_{j} \dot{x}_{j}+\omega_{j}^{2} x_{j}=\vec{\phi}_{j}^{\top} \vec{u} \\
\vec{y}_{j}=\vec{\gamma}_{j} x_{j}+\vec{\eta}_{j} \dot{x}_{j}
\end{array}
$$

where $\zeta_{j}$ is the damping ratio of the mode. Damping ratios less than one correspond to underdamped modes, i.e. modes of vibration. Due to the lack of fluid damping in space and the light weight of space structures, we will be primarily concerned with underdamped systems.

When damping is introduced into a model as in (2.12), we are implicitly assuming that the form of the system's damping matrix $D$ is such that it will be simultaneously diagonalized along with the mass and stiffness matrices in (2.11). As noted by Garvey et al. in [5] there is considerable disagreement about what to call the type of damping that adheres to this constraint on the damping matrix. Common names for this damping model are sometimes conflated with the more restrictive Rayleigh damping condition. In this work we will refer to damping that satisfies this constraint as modal damping. 


\subsection{Conversion to First-Order Form}

In control engineering, first-order state space is the standard form in which problems are cast:

$$
\begin{aligned}
& \dot{\vec{x}}=A \vec{x}+B \vec{u} \\
& \vec{y}=C \vec{x}+D \vec{u}
\end{aligned}
$$

where $\vec{u}$ and $\vec{y}$ are the input and output respectively, $\vec{x}$ is the system state, $A$ is the state transition matrix, and $B$ and $C$ are the actuator and sensor matrices. The feedforward matrix $D$ (which is not a damping matrix) is included in equation (2.13) for completeness but will not be utilized in this work.

Given the second-order matrix ordinary differential equation (2.2) we can convert it to a first-order form by first dividing through by the mass matrix and then combining the node displacements and velocities into a block-form state vector as in the following block matrix equation:

$$
\begin{array}{r}
\dot{\vec{x}}=\left[\begin{array}{c}
\dot{\vec{q}} \\
\ddot{\vec{q}}
\end{array}\right]=\left[\begin{array}{cc}
0 & I \\
-M^{-1} K & -M^{-1} D
\end{array}\right]\left[\begin{array}{c}
\vec{q} \\
\dot{\vec{q}}
\end{array}\right]+\left[\begin{array}{c}
0 \\
M^{-1} F
\end{array}\right] \vec{u} \\
\vec{y}=\left[\begin{array}{ll}
G & H
\end{array}\right]\left[\begin{array}{c}
\vec{q} \\
\dot{\vec{q}}
\end{array}\right]
\end{array}
$$

where the square matrices 0 and $I$ are zero and identity matrices respectively that conform to the dimensions of this block matrix. Similarly, the harmonic oscillator in equation (2.12) can be cast in a first-order form:

$$
\begin{array}{r}
{\left[\begin{array}{c}
\dot{x}_{j} \\
\ddot{x}_{j}
\end{array}\right]=\left[\begin{array}{cc}
0 & 1 \\
-\omega_{j}^{2} & -2 \zeta_{j} \omega_{j}
\end{array}\right]\left[\begin{array}{c}
x_{j} \\
\dot{x}_{j}
\end{array}\right]+\left[\begin{array}{c}
\overrightarrow{0}^{\top} \\
\vec{\phi}_{j}^{\top}
\end{array}\right] \vec{u}} \\
\vec{y}=\left[\begin{array}{ll}
\vec{\gamma}_{j} & \vec{\eta}_{j}
\end{array}\right]\left[\begin{array}{c}
x_{j} \\
\dot{x}_{j}
\end{array}\right]
\end{array}
$$


where the $\overrightarrow{0}^{\top}$ in the rightmost block matrix of the above is a zero column vector of conformable size. Utilizing (2.15), a system in a second-order modal form can be rewritten as a first-order state space form in which the state-transition matrix $A$ is block-diagonal, with each entry a two-by-two matrix. Furthermore, these blocks can be arranged so that the $j$ th structural mode of the system corresponds to the $j$ th two-by-two block on the diagonal.

\section{$2.4 \quad$ First-Order Equivalent Representations}

As we have already seen through the example of the second-order modal form of a model, state space representations of linear systems are not unique. Two equivalent systems will have identical output values for a given input value and initial conditions - the only difference is in the coordinates used for viewing the internal system state. Note that two first-order system forms are equivalent if they are related to each other by an invertible linear coordinate transformation. Any equivalent representation of a given state space model as in (2.13) can be reached by transforming the original model as follows:

$$
\begin{array}{r}
\dot{\vec{x}}_{T}=\left(T A T^{-1}\right) \vec{x}_{T}+(T B) \vec{u} \\
\vec{y}=\left(C T^{-1}\right) \vec{x}_{T}+D \vec{u}
\end{array}
$$

where $T$ is any invertible coordinate transformation matrix and $\vec{x}_{T}$ is the system state in the coordinate system induced by $T$. For example, as described earlier we know that the first-order form in (2.14) has an equivalent representation that is block-diagonal, with each block of taking the form of the state transition matrix in (2.15). It then follows that some coordinate transformation matrix $T$ relates these two forms via (2.16). Although we know such a transformation matrix exists, computing it is another matter - even when a matrix is found that correctly 
converts the state transition matrix $A$ to the desired block-diagonal form, there is no guarantee that the actuator and sensor matrices $B$ and $C$ will be transformed appropriately as well.

\subsection{Modalization of Models in First-Order Form}

The eigenvalue decomposition applied to a model in first-order form is another example of both an equivalent system and a modalization procedure. Each mode of vibration in a second-order system gives rise to a pair of complex-valued eigenvalues of the same system in first-order form, with the system state becoming complex-valued as well. We will begin our discussion of modal forms for first-order systems by re-examining (2.15) and noting that the eigenvalues corresponding to the $j$ th mode are the roots of the characteristic polynomial:

$$
\lambda_{j}^{2}+2 \zeta_{j} \omega_{j} \lambda_{j}+\omega_{j}^{2} \lambda_{j}=0
$$

where $2 \zeta_{j} \omega_{j}$ is the negative of the trace of the matrix, and $\omega_{j}^{2}$ is its determinant. The complex eigenvalues are solved for using the quadric formula to be:

$$
\begin{aligned}
& \lambda_{j, 1}=-\zeta_{j} \omega_{j}+\hat{i} \omega_{j} \sqrt{1-\zeta^{2}} \\
& \lambda_{j, 2}=-\zeta_{j} \omega_{j}-\hat{i} \omega_{j} \sqrt{1-\zeta^{2}}
\end{aligned}
$$

where $\hat{i}$ is the imaginary unit. The eigenvalues of the state transition matrix in (2.14) will be the union of the eigenvalues of the individual modes, where the eigenvalues of the $j$ th mode have the form shown in (2.18). Note that the natural frequency $\omega_{j}$ is the absolute value of either $\lambda_{j}$ since:

$$
\left|\lambda_{j}\right|=\sqrt{\left(-\zeta_{j} \omega_{j}\right)^{2}+\left(\omega_{j} \sqrt{1-\zeta^{2}}\right)^{2}}=\omega_{j} \sqrt{\zeta_{j}^{2}+\left(1-\zeta_{j}^{2}\right)}=\omega_{j}
$$

It is tempting to think that one can alter the damping ratio and natural frequency of the $j$ th mode via the eigenvalue decomposition, using (2.18) and (2.19). 
For example, after computing the eigenvalue decomposition, the desired eigenvalues could be substituted for the old ones, and the system back-transformed to the original real-valued coordinate system. Note, however, that doing so is not equivalent to adding modal damping via the second-order modal decomposition, and unrealistic system dynamics can result. Continuing, we will derive a process for obtaining the usual second-order form with modal damping as in (2.12) for any single-input system $(A, B, C)$ in which all complex eigenvalues of $A$ come in unique conjugate pairs. The general method is a variation on the ideas presented by Patil in [20]. We will start by showing how to obtain a real-valued modal form for any such system.

First we will note that any two-by-two real-valued matrix $J$ that takes the following form:

$$
J=\left[\begin{array}{cc}
\alpha & -\beta \\
\beta & \alpha
\end{array}\right]
$$

will have eigenvalues:

$$
\kappa_{1,2}=\alpha \pm \hat{i} \beta
$$

with corresponding eigenvectors:

$$
\vec{u}_{1,2}=\left[\begin{array}{c}
1 \\
\mp \hat{i}
\end{array}\right] .
$$

We can now calculate a coordinate transformation mapping a real matrix $A$ with conjugate pairs of complex eignvalues to a real-valued form that is block-diagonal, with each block of the form (2.20). If we are given an eigendecomposition of $A$, then for each pair of complex conjugate eigenvalues we can scale the corresponding eigenvectors to ensure they also come in conjugate pairs. After doing so we can use (2.22) to calculate a real-valued transformation mapping $A$ to a block-diagonal real matrix with each block of the form of $J$ in (2.20). Observe that the matrix 
$U$ of the eigenvectors $\vec{u}_{1,2}$ of $J$ and its inverse $U^{-1}$ are:

$$
\begin{gathered}
U=\left[\begin{array}{cc}
1 & 1 \\
-\hat{i} & \hat{i}
\end{array}\right] \\
U^{-1}=\left[\begin{array}{cc}
1 & \hat{i} \\
1 & -\hat{i}
\end{array}\right] \frac{1}{2} .
\end{gathered}
$$

Examining $U^{-1}$, we see that multiplying any matrix $W$, formed from two column vectors that are each other's complex conjugates by $U^{-1}$, is equivalent to forming a new matrix from the real and imaginary parts of one column of $W$. For example, let $\vec{p}$ and $\vec{q}$ be real-valued vectors so that the first column of $W$ is the vector quantity $(\vec{p}-\hat{i} \vec{q})$, and then multiply $W$ by $U^{-1}$ as follows:

$$
\begin{aligned}
W U^{-1} & =\left[\begin{array}{ll}
\vec{p}-\hat{i} \vec{q} & \vec{p}+\hat{i} \vec{q}
\end{array}\right]\left[\begin{array}{cc}
1 & \hat{i} \\
1 & -\hat{i}
\end{array}\right] \frac{1}{2} \\
& =\left[\begin{array}{ll}
\vec{p}+\vec{p} & \vec{q}+\vec{q}
\end{array}\right] \frac{1}{2}=\left[\begin{array}{ll}
\vec{p} & \vec{q}
\end{array}\right] .
\end{aligned}
$$

If we let $\tilde{W}$ be a matrix whose columns are eigenvectors of a real state-transition matrix $A$, ordered so that conjugate pairs of eigenvectors are adjacent, and let $\tilde{U}^{-1}$ be a matrix that is block-diagonal, with a two-by-two block identical to $U^{-1}$ at each location corresponding to a pair of complex-conjugate eigenvectors, then a real-valued coordinate transformation matrix $T_{R}$ mapping the system to a modal form is:

$$
T_{R}=\left(\tilde{W} \tilde{U}^{-1}\right)^{-1}
$$

Using (2.24) the matrix quantity $\left(\tilde{W} \tilde{U}^{-1}\right)$ can be created filling in its columns a pair at a time for each pair of complex eigenvectors, with the first column being the real part and the second column the imaginary part of an eigenvector of one of the complex eigenvalues in each conjugate pair. This can be done for all the 
eigenvalues whose imaginary part is positive, for example. Doing so also allows us to drop the requirement of finding an entire eigendecomposition.

Given a single-input system that has been transformed to a real-valued modal form, with the block-diagonal consisting entirely of two-by-two blocks as in (2.20), we can take the process one step further and compute a modal form equivalent to the usual second-order one. First observe that an eigenvector $\vec{v}$ of the two-bytwo matrix in (2.15) can be written in terms of its corresponding eigenvalue $\lambda$ as follows:

$$
\vec{v}=\left[\begin{array}{l}
1 \\
\lambda
\end{array}\right]
$$

so that a matrix $V$ of such eigenvectors (as would be computed in an eigendecomposition) could be:

$$
V=\left[\begin{array}{cc}
1 & 1 \\
\lambda_{1} & \lambda_{2}
\end{array}\right] .
$$

Using (2.27) we will compute a coordinate transformation that takes a singleinput system with two-by-two state transition matrix of the form in (2.20) to the form of a harmonic oscillator, as in (2.15). The obvious extension of this is our desired method for conversion of a block-diagonal system whose block diagonal consists entirely of two-by-two blocks as in (2.20) to a modal form that is equivalent to the usual second-order one, one block at a time.

Let $(A, B, C)$ be a single-input system where $A$ takes the exact form of the two-by-two matrix $J$ in (2.20) and has $\kappa_{1,2}$ as its eigenvalues as in (2.21). Let the matrix $V$ in $(2.27)$ be defined so that:

$$
\lambda_{1,2}=\kappa_{1,2}=\alpha \pm \hat{i} \beta .
$$

Applying (2.24) with $V$ substituted for $W$, we get that the matrix quantity $\left(V U^{-1}\right)$ is real-valued, with its first column being the real part of the second 
column of (2.27) and the second column its imaginary part, so that:

$$
V U^{-1}=\left[\begin{array}{cc}
1 & 0 \\
\operatorname{Real}\left(\lambda_{2}\right) & \operatorname{Imag}\left(\lambda_{2}\right)
\end{array}\right]=\left[\begin{array}{cc}
1 & 0 \\
\alpha & -\beta
\end{array}\right] .
$$

In order for the transformation given by $\left(V U^{-1}\right)$ to convert our system $(A, B, C)$ to the form in $(2.15)$, it would have to be the case that:

$$
\left(V U^{-1}\right) B=\left[\begin{array}{l}
0 \\
\phi
\end{array}\right]
$$

or equivalently:

$$
\left[\begin{array}{cc}
1 & 0 \\
\alpha & -\beta
\end{array}\right]\left[\begin{array}{c}
b_{1} \\
b_{2}
\end{array}\right]=\left[\begin{array}{c}
b_{1} \\
\alpha b_{1}-\beta b_{2}
\end{array}\right]=\left[\begin{array}{l}
0 \\
\phi
\end{array}\right]
$$

where $\phi$ is the actuator input term as in (2.15) and is real-valued as opposed to vector-valued since we assumed a single-input system, and $b_{i}$ are the components of $B$, which in this case is a column vector for the same reason. Note that (2.30) is easily resolved if both $b_{i}$ are zero, since this implies $\phi$ is zero and the system is uncontrollable. Similarly the problem can be solved via a simple scaling if $b_{1}$ is zero. We will assume that at least one $b_{i}$ is non-zero and seek a new transformation to sidestep the constraints posed by (2.31).

Fortunately, since all matrices of the form in (2.20) will have the same eigenvectors, namely (2.22), any two such matrices will commute. This implies that $A$ is invariant under any coordinate change by such a matrix, so we can extend the transformation in (2.30) to include such a coordinate transformation by a matrix $R$ as follows:

$$
\left(V U^{-1}\right) R B=\left[\begin{array}{l}
0 \\
\phi
\end{array}\right]
$$


Equivalently:

$$
R\left[\begin{array}{l}
b_{1} \\
b_{2}
\end{array}\right]=\left(V U^{-1}\right)^{-1}\left[\begin{array}{l}
0 \\
\phi
\end{array}\right]=\frac{1}{\beta}\left[\begin{array}{cc}
\beta & 0 \\
\alpha & -1
\end{array}\right]\left[\begin{array}{c}
0 \\
\phi
\end{array}\right]=\frac{1}{\beta}\left[\begin{array}{c}
0 \\
-\phi
\end{array}\right]
$$

so that $R$ takes the form:

$$
R=\left[\begin{array}{cc}
-b_{2} & b_{1} \\
-b_{1} & -b_{2}
\end{array}\right] \frac{\phi}{\beta\left(b_{1}^{2}+b_{2}^{2}\right)}
$$

It follows that the entire coordinate transformation $T$ represented by the matrix quantity $\left(V U^{-1} R\right)$ is:

$$
\begin{aligned}
T=\left(V U^{-1}\right) R & =\left[\begin{array}{cc}
1 & 0 \\
\alpha & -\beta
\end{array}\right]\left[\begin{array}{cc}
-b_{2} & b_{1} \\
-b_{1} & -b_{2}
\end{array}\right] \frac{\phi}{\beta\left(b_{1}^{2}+b_{2}^{2}\right)} \\
& =\left[\begin{array}{cc}
-b_{2} & b_{1} \\
\beta b_{1}-\alpha b_{2} & \alpha b_{1}+\beta b_{2}
\end{array}\right] \frac{\phi}{\beta\left(b_{1}^{2}+b_{2}^{2}\right)}
\end{aligned}
$$

Note that if the first-order system $(A, B, C)$ with $A$ identical to $J$ in $(2.20)$ is instead assumed to be multi-input, but is known to be constrained such that it has an equivalent second-order form with modal damping, we can compute $T$ in (2.35) using any non-zero column of $B$ to obtain the $b_{i}$. In this case $T$ will transform the system to a second-order equivalent form because the constraint eliminated the extra degrees of freedom. There are some instances, however, when we may want to obtain an approximation for a second-order equivalent form of $(A, B, C)$ with modal damping when none exists. For example, we may have applied a model reduction technique to the first-order form of a secondorder system, and in the process rendered it no longer second-order equivalent. The desire to avoid this situation is one reason why there is interest in model reduction techniques that preserve second-order forms, as in [22]. 
To assess the "damage" done to a formerly second-order system with modal damping, we can compute an approximate version of $T$ in which the matrix $R$ is defined to minimize the ratio of the 2-norms of the two rows of the transformed actuator matrix, $(T B)$. A pair of $R$ matrices, $R_{1,2}$, one of which will maximize the ratio of the norms, with the other minimizing it were found to be:

$$
\begin{array}{r}
R_{1,2}=\left[\begin{array}{cc}
\cos \theta_{1,2} & -\sin \theta_{1,2} \\
\sin \theta_{1,2} & \cos \theta_{1,2}
\end{array}\right] \\
\theta_{1}=\frac{1}{2} \arg \left(\vec{b}_{1}^{\top}-\hat{i} \vec{b}_{2}^{\top}\right)\left(\vec{b}_{1}-\hat{i} \vec{b}_{2}\right) \\
\theta_{2}=\theta_{1}+\frac{\pi}{2}
\end{array}
$$

where the $\vec{b}_{i}^{\top}$ are the two rows of $B$.

If the desired form of the transformed sensor matrix $\left(C T^{-1}\right)$ is similarly known to measure only velocity states (or if it measures only position states, swap the two columns of $C$ before continuing) we can alter (2.36) to give the option of accounting for this, by forming a matrix $B^{\prime}$ that is substituted for $B$ when computing $R_{1,2}$ :

$$
B^{\prime}=\left[\begin{array}{ll}
\mu B & \nu C^{\top}
\end{array}\right]
$$

where $\mu$ and $\nu$ are weighting parameters.

In closing, we will note that although the formulas presented here relied on the modes of interest having pairs of complex conjugate eigenvalues, pairs of stable real eigenvalues can also be converted to modal blocks, but with ambiguity present when more than one pair of real eigenvalues can be formed. When a system has a single real eigenvalue, however, we must either leave it be as we partially modalize the system, or alternatively perform modal truncation model reduction on this single state to eliminate it from the model. 


\section{Chapter 3}

\section{Model Reduction}

\subsection{Modal Truncation}

Modal truncation is a model reduction technique that has the advantage of being easily understood and implemented. First a system is transformed to a modal form. This could be a real-valued modal representation of a system in first-order form, the usual second-order modal form of a finite element model with modal damping, or even a complex-valued modal form, amongst other options. After transforming the system, the designer chooses which modes to truncate from the model. The rows and columns of the system matrices whose dynamical states correspond to these modes are then truncated from the model. Consider the example below in which the following two-mode system in second-order modal 
form has its second mode truncated:

$$
\begin{gathered}
{\left[\begin{array}{c}
\ddot{x_{1}} \\
\ddot{x_{2}}
\end{array}\right]+\left[\begin{array}{cc}
2 \zeta_{1} \omega_{1} & 0 \\
0 & 2 \zeta_{2} \omega_{2}
\end{array}\right]\left[\begin{array}{c}
\dot{x_{1}} \\
\dot{x_{2}}
\end{array}\right]+\left[\begin{array}{cc}
\omega_{1}^{2} & 0 \\
0 & \omega_{2}^{2}
\end{array}\right]\left[\begin{array}{c}
x_{1} \\
x_{2}
\end{array}\right]=\left[\begin{array}{c}
\vec{\phi}_{1}^{\top} \\
\vec{\phi}_{2}^{\top}
\end{array}\right] \vec{u}} \\
\vec{y}=\left[\begin{array}{ll}
\vec{\gamma}_{1} & \vec{\gamma}_{2}
\end{array}\right]\left[\begin{array}{c}
x_{1} \\
x_{2}
\end{array}\right]+\left[\begin{array}{ll}
\vec{\eta}_{1} & \vec{\eta}_{2}
\end{array}\right]\left[\begin{array}{c}
\dot{x}_{1} \\
\dot{x}_{2}
\end{array}\right]
\end{gathered}
$$

resulting in a single harmonic oscillator corresponding to the first mode:

$$
\begin{array}{r}
\ddot{x}_{1}+2 \zeta_{1} \omega_{1} \dot{x}_{1}+\omega_{1}^{2} x_{1}=\vec{\phi}_{1}^{\top} \vec{u} \\
\vec{y}=\vec{\gamma}_{1} x_{1}+\vec{\eta}_{1} \dot{x}_{1} .
\end{array}
$$

Perhaps somewhat intuitively, usually the modes with the highest natural frequencies are truncated first. This makes sense because modal damping for a mode is proportional to its natural frequency. The contribution of highly-damped modes to the overall system dynamics is usually minimal due to their high decay rates. The designer may also consider truncating modes from a model that have resonances occurring at frequencies far outside of the range of operating frequencies of the plant. Additionally, the frequency-response information of a model may be considered to help either a designer or an automated model reduction algorithm distinguish between high-gain and low-gain modes, with the goal of preserving the dominant system dynamics. Specialized algorithms for computing the eigenvalues of dominant modes, as in [10] are an interesting example.

\subsection{Controllability and Observability Gramians}

One drawback of "blind" modal truncation methods (i.e. approaches that do not incorporate frequency-response information into the model reduction process) is that they do not account for the effects of the sensors and actuators represented 
by the $B$ and $C$ matrices of a first-order representation. For example, there may be some modes that are very difficult to excite due to the configuration of the actuators, or others that make a negligible contribution to the output signal based on the placement of the sensors. Such modes would be referred to as nearly uncontrollable/observable respectively. Information regarding the controllability and observability of a system's states is conveniently encapsulated by a pair of matrices known as the controllability and observability gramians. These matrices are defined for any first-order representation of a stable linear system, and are symmetric and positive-semidefinite. Specifically, for a given time interval from zero to $\tau$ and system state $\vec{x}$, the controllability and observability gramians answer the following questions respectively: 1) how much signal energy is required at the control input $\vec{u}$ to drive the system state from the coordinate origin to $\vec{x}$, over the time interval from zero to $\tau ; 2$ ) how much signal energy passes through the output $\vec{y}$, over the time interval from zero to $\tau$, given an initial state of $\vec{x}$ and no control input? As in [12], we will define the controllability and observability gramians for a system $(A, B, C)$ over a time interval from zero to $\tau$ as follows:

$$
\begin{aligned}
& L_{c}=\int_{0}^{\tau} e^{A t} B B^{\top} e^{A^{\top} t} d t \\
& L_{o}=\int_{0}^{\tau} e^{A^{\top} t} C^{\top} C e^{A t} d t .
\end{aligned}
$$

According to [13] the input and output signal energies are:

$$
\begin{aligned}
& E_{\vec{u}}=\vec{x}^{\top} L_{c}^{-1} \vec{x} \\
& E_{\vec{y}}=\vec{x}^{\top} L_{o} \vec{x} .
\end{aligned}
$$


Note that when $L_{c}$ is not full-rank, the system is not controllable, and the gramian is not invertible. If we view attempts to compute the inverse of $L_{c}$ from an eigenvalue-eigenvector perspective, we see that any eigenvalues of the gramian that are zero will have corresponding eigenvectors that represent subspaces of states that are unreachable - that is, it would require an infinite amount of input signal energy to force the system into such a state. Similarly, when $L_{o}$ is not full-rank, the system is not observable, and there exist subspaces of states (which are formed from the eigenvectors of $L_{o}$ that correspond to null eigenvalues) whose signal energies will not contribute to the output signal energy .

To solve for the minimum required input signal energy to reach a state $\vec{x}$ and maximum possible output signal energy obtainable from $\vec{x}$ we carry out the computation of the gramians in the limit as $\tau$ goes to infinity. This results in the pair of infinite gramians $W_{c}$ and $W_{o}$ :

$$
\begin{aligned}
& W_{c}=\lim _{\tau \rightarrow \infty} \int_{0}^{\tau} e^{A t} B B^{\top} e^{A^{\top} t} d t \\
& W_{o}=\lim _{\tau \rightarrow \infty} \int_{0}^{\tau} e^{A^{\top} t} C^{\top} C e^{A t} d t .
\end{aligned}
$$

As noted in [12], the infinite gramians can also be computed by solving the Lyapunov matrix equation:

$$
P X+X P^{\top}+Q=0
$$

as shown in (3.10) and (3.11):

$$
\begin{aligned}
& A W_{c}+W_{c} A^{\top}+B B^{\top}=0 \\
& A^{\top} W_{o}+W_{o} A+C^{\top} C=0 .
\end{aligned}
$$


Note that we can also use the Lyapunov matrix equation to show how the infinite gramians transform when the state space representation is written in a different coordinate system as in equation (2.16). Using (2.16) the Lyapunov matrix equations for the transformed gramians, $W_{o}^{\prime}$ and $W_{c}^{\prime}$, are:

$$
\begin{gathered}
T A T^{-1} W_{c}^{\prime}+W_{c}^{\prime} T^{-\top} A^{\top} T^{\top}+T B B^{\top} T^{\top}=0 \\
T^{-\top} A^{\top} T^{\top} W_{o}^{\prime}+W_{o}^{\prime} T A T^{-1}+T^{-\top} C^{\top} C T^{-1}=0 .
\end{gathered}
$$

To solve for $W_{c}^{\prime}$ and $W_{o}^{\prime}$ we rederive (3.12) and (3.13), starting from the Lyapunov equations in the previous coordinate system, (3.10) and (3.11), for which we have the solutions $W_{c}$ and $W_{o}$ respectively:

$$
\begin{array}{r}
0=T(0) T^{\top}=T\left(A W_{c}+W_{c} A^{\top}+B B^{\top}\right) T^{\top} \\
=T A W_{c} T^{\top}+T W_{c} A^{\top} T^{\top}+T B B^{\top} T^{\top} \\
=T A\left(T^{-1} T\right) W_{c} T^{\top}+T W_{c}\left(T^{\top} T^{-\top}\right) A^{\top} T^{\top}+T B B^{\top} T^{\top} \\
=T A T^{-1}\left(T W_{c} T^{\top}\right)+\left(T W_{c} T^{\top}\right) T^{-\top} A^{\top} T^{\top}+T B B^{\top} T^{\top} \\
0=T^{-\top}(0) T^{-1}=T^{-\top}\left(A^{\top} W_{o}+W_{o} A+C^{\top} C\right) T^{-1} \\
=T^{-\top} A^{\top} W_{o} T^{-1}+T^{-\top} W_{o} A T^{-1}+T^{-\top} C^{\top} C T^{-1} \\
=T^{-\top} A^{\top}\left(T^{\top} T^{-\top}\right) W_{o} T^{-1}+T^{-\top} W_{o}\left(T^{-1} T\right) A T^{-1}+T^{-\top} C^{\top} C T^{-1} \\
=T^{-\top} A^{\top} T^{\top}\left(T^{-\top} W_{o} T^{-1}\right)+\left(T^{-\top} W_{o} T^{-1}\right) T A T^{-1}+T^{-\top} C^{\top} C T^{-1}
\end{array}
$$

and notice that:

$$
\begin{gathered}
W_{c}^{\prime}=T W_{c} T^{\top} \\
W_{o}^{\prime}=T^{-\top} W_{o} T^{-1} .
\end{gathered}
$$




\subsection{Balanced Truncation}

When a state space representation of a linear system has controllability and observability gramians that are equal and diagonal, with the diagonal terms ordered from greatest to least, the representation is said to be balanced. Because the gramians are diagonal, the choice of coordinates in a balanced representation is such that the system's states are eigenvectors of the gramians. The diagonal entries, $\sigma_{i, i}$, of the balanced gramians are positive, and are called Hankel singular values (HSV's). The Hankel singular values carry with them a useful interpretation - small HSV's correspond to states that are relatively uncontrollable and unobservable, that is, they correspond to system states that require large signal input energy to excite, and which return very little of this energy to the output signal. It thus makes sense to truncate such states from a model, since they contribute very little to the system dynamics. As described by Mehiel in [16], a common procedure for determining how many states to truncate involves plotting the HSV's on a log scale and looking for a "knee in the curve" that separates larger magnitude HSV's from smaller ones. Examining figure 3.1 for example, we see that about eight states would be kept in the model, corresponding to the eight HSV's above the "knee."

To compute a balancing transformation using the infinite gramians, we seek a coordinate transformation matrix $T$ satisfying:

$$
T W_{c} T^{\top}=T^{-\top} W_{o} T^{-1}
$$

with the additional constraint that the transformed gramians are diagonal. If we are given a system $(A, B, C)$ and its gramians $W_{c}$ and $W_{o}$ that are known to be positive-definite - i.e. the system is stable, controllable and observable, then finding a balancing transformation from the gramians is not too difficult. 


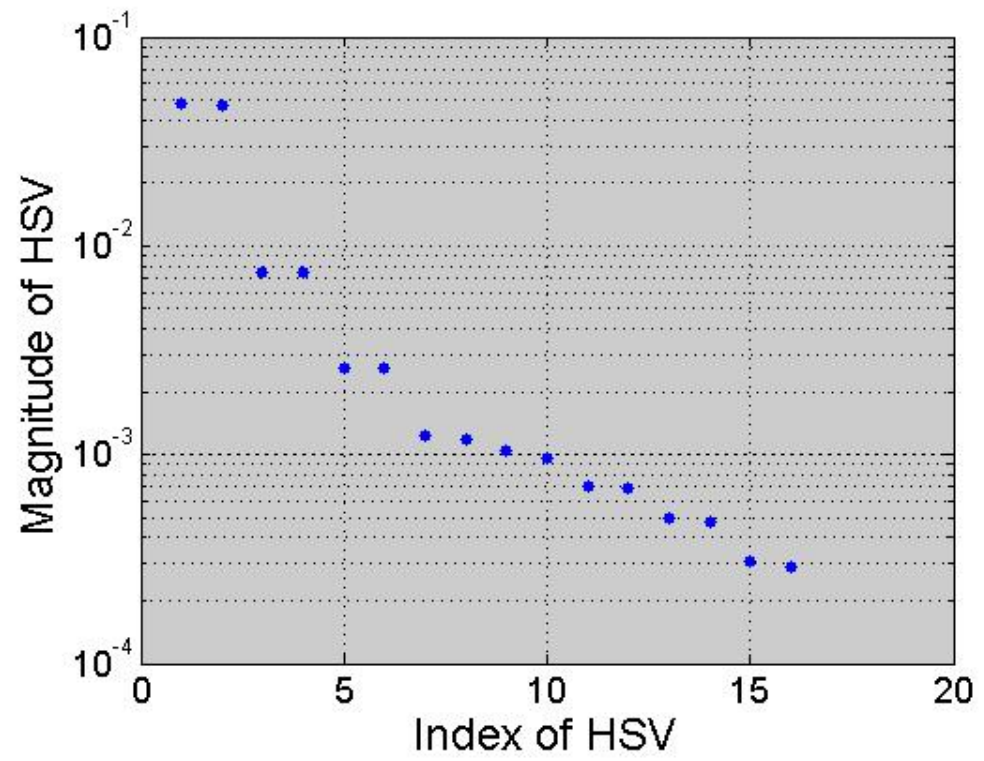

Figure 3.1: Log-scale plot of Hankel singular values for an 8-mode model. The Hankel Singular Values of the balanced gramians are plotted on $\log$ scale to find a "knee in the curve," below which the states with small HSV's are truncated.

Gramian-free methods such as the one presented in [12] are recommended in practice, however - the following derivation is for illustrative purposes only.

The main idea of the computation involves finding the singular value decomposition of a matrix quantity formed as the product of two matrices. Let $P$ and $Q$ be two matrices, and let $U$ and $V$ be matrices whose column vectors are the left and right singular vectors of the matrix quantity $(P Q)$ so that the singular value decomposition of $(P Q)$ is:

$$
(P Q)=U \Sigma V^{\top} .
$$

Then each of the possible products of $(P Q)$ with its transpose are diagonalizable by $U$ or $V$ and the diagonal matrices are equal to the square of the diagonal 
matrix of singular values, $\Sigma^{2}$ :

$$
\begin{gathered}
U^{\top}(P Q)(P Q)^{\top} U=U^{\top}\left(U \Sigma V^{\top}\right)\left(V \Sigma U^{\top}\right) U=\Sigma^{2} \\
V^{\top}(P Q)^{\top}(P Q) V=V^{\top}\left(V \Sigma U^{\top}\right)\left(U \Sigma V^{\top}\right) V=\Sigma^{2} .
\end{gathered}
$$

If we suppose $P$ and $Q$ are symmetric, then we obtain:

$$
\begin{gathered}
U^{\top} P Q^{2} P U=\Sigma^{2} \\
V^{\top} Q P^{2} Q V=\Sigma^{2} .
\end{gathered}
$$

Observing this, we let $P$ and $Q$ be the symmetric square roots of the infinite observability and controllability gramians, so that (3.21) becomes:

$$
\begin{gathered}
U^{\top} W_{o}^{1 / 2} W_{c} W_{o}^{1 / 2} U=\Sigma^{2} \\
V^{\top} W_{c}^{1 / 2} W_{o} W_{c}^{1 / 2} V=\Sigma^{2} .
\end{gathered}
$$

Notice that (3.22) can be interpreted as a method for transforming the gramians $W_{c}$ and $W_{o}$ to be equal and diagonal by two separate bilinear coordinate transformations since:

$$
\begin{gathered}
U^{\top} W_{o}^{1 / 2}=\left(W_{o}^{1 / 2} U\right)^{\top} \\
\left(W_{c}^{1 / 2} V\right)^{\top}=V^{\top} W_{c}^{1 / 2} .
\end{gathered}
$$

The only remaining issue to resolve is that the coordinate transformation matrices for the bilinear coordinate transformations in (3.22) need to be inverses of each other, as in equation (3.18). Fortunately, this problem has a resolution - it relies on the fact that the singular value decomposition can be used to compute the matrix inverse. If we apply this technique for computing a matrix inverse to the singular value decomposition of the matrix quantity in equation (3.19) with $P$ and $Q$ being the symmetric square roots of $W_{o}$ and $W_{c}$ respectively, we get:

$$
\left(W_{o}^{1 / 2} W_{c}^{1 / 2}\right)^{-1}=V \Sigma^{-1} U^{\top} .
$$


Notice then that:

$$
\begin{aligned}
I=(I)(I) & =\left(W_{c}^{1 / 2} W_{c}^{-1 / 2}\right)\left(W_{o}^{-1 / 2} W_{o}^{1 / 2}\right)=W_{c}^{1 / 2}\left(W_{o}^{1 / 2} W_{c}^{1 / 2}\right)^{-1} W_{o}^{1 / 2} \\
& =W_{c}^{1 / 2}\left(V \Sigma^{-1} U^{\top}\right) W_{o}^{1 / 2}=\left(W_{c}^{1 / 2} V \Sigma^{-1 / 2}\right)\left(\Sigma^{-1 / 2} U^{\top} W_{o}^{1 / 2}\right)
\end{aligned}
$$

where $I$ is an appropriately-sized identity matrix. Notice that the two matrix quantities in the last equality in (3.25) can be obtained by appropriately multiplying the transformation matrices of the bilinear coordinate transformations in (3.22) by the square root of the multiplicative inverse of the $\Sigma$ matrix. It follows that the transformation matrix (and its inverse) that place a given first-order state space representation into balanced coordinates can be computed as:

$$
\begin{gathered}
T=\Sigma^{-1 / 2} U^{\top} W_{o}^{1 / 2} \\
T^{-1}=W_{c}^{1 / 2} V \Sigma^{-1 / 2}
\end{gathered}
$$

so the gramians are transformed as:

$$
\begin{array}{r}
T W_{c} T^{\top}=\Sigma^{-1 / 2} U^{\top} W_{o}^{1 / 2} W_{c} W_{o}^{1 / 2} U \Sigma^{-1 / 2}=\Sigma^{-1 / 2} \Sigma^{2} \Sigma^{-1 / 2}=\Sigma \\
T^{-\top} W_{o} T^{-1}=\Sigma^{-1 / 2} V^{\top} W_{c}^{1 / 2} W_{o} W_{c}^{1 / 2} V \Sigma^{-1 / 2}=\Sigma^{-1 / 2} \Sigma^{2} \Sigma^{-1 / 2}=\Sigma .
\end{array}
$$

As (3.27) shows, the Hankel singular values are actually the singular values of the matrix quantity $\left(W_{o}^{1 / 2} W_{c}^{1 / 2}\right)$.

The Hankel singular values can be used in simple calculations of error bounds for approximate models generated via balanced model reduction methods. As an example, we will consider an error bound proved by Glover in [6] for balanced truncation model reduction. Let $\sigma_{i, i}$ denote the $i$ th Hankel singular value of an $n$-state system $(A, B, C)$, and let $(\tilde{A}, \tilde{B}, \tilde{C})$ be a reduced-order model obtained by truncating the last $k$ HSV's (with $k$ less than $n$ ). Specifically, Glover's error bound is a bound on the infinity norm of the difference of the transfer function matrices corresponding to the full-order and reduced-order models. Let $H(s)$ and $\tilde{H}(s)$ be the transfer function matrices of the system and a stable reduced-order 
model obtained by truncating balanced states corresponding to the last $k$ HSV's of the gramians. As noted by Mehiel [16], these transfer function matrices can be computed as follows:

$$
\begin{gathered}
H(s)=C(s I-A)^{-1} B \\
\tilde{H}(s)=\tilde{C}(s I-\tilde{A})^{-1} \tilde{B} .
\end{gathered}
$$

The error bound is then defined as:

$$
\|H(s)-\tilde{H}(s)\|_{\infty} \leq 2 \sum_{i=n-k+1}^{n} \sigma_{i, i} .
$$

One key quality of reduced order models computed via modal truncation, as opposed to a balanced reduction, is that the modes the designer chooses to retain in the modal approach are preserved completely - there is no approximation error with respect to these modes individually. It is this property that guarantees the preservation of system stability in a reduced-order modal model. We have not discussed the stability of truncated balanced models, but fortunately there is a proof by Pernebo and Silverman [21] that guarantees the stability of such a model, based on one condition: if two or more Hankel singular values of the full-order system are equal, then either all or none of the balanced states corresponding to these HSVs must be truncated when forming the reduced-order model. The next section focuses on addressing the issues posed by the degradation of the system modes in balanced truncation.

\subsection{Modes in the Balanced Truncation}

If we follow the standard balanced truncation algorithm, we don't have control over which modes we keep in the model - the algorithm eliminates states with the smallest corresponding Hankel singular values first, and we do not know a 
priori the relationships between the balanced states and the structural modes. Moreover, truncating the rows and columns corresponding to a single balanced state from the model introduces approximation error into all modes that are linked to that state - the approximate model will have altered eigenvalues and resonant frequencies, and every mode in the model can potentially be impacted.

One approach to establishing the relationship between the states of the balanced representation and the structural modes of the system is to back-project the image of the truncated balanced model into the modal coordinates of the full-order model, and then compute an approximation error with respect to each mode individually. Let $A_{m}$ be the state-transition matrix of a first-order state space with $n$ states, in modal form, so that it is block diagonal with entries as in equation (2.12), with the $j$ th two-by-two block corresponding to the $j$ th structural mode of the system. Let $T$ be the coordinate transformation matrix that converts this state space to an equivalent balanced representation with state transition matrix $A_{b}$. We will view $T$ and $T^{-1}$ as block matrices of row and column vectors respectively as follows:

$$
\begin{array}{r}
T=\left[\begin{array}{c}
\vec{u}_{1}^{\top} \\
\vdots \\
\vec{u}_{n}^{\top}
\end{array}\right] \\
T^{-1}=\left[\begin{array}{lll}
\vec{v}_{1} & \ldots & \vec{v}_{n}
\end{array}\right] .
\end{array}
$$

where $\vec{u}_{i}^{\top}$ is the $i$ th row of $T$ and $\vec{v}_{i}$ is the $i$ th column of $T^{-1}$. Let $\tilde{A}_{b}$ be the balanced reduced-order model's state-transition matrix, obtained by removing the states corresponding to the $k$ smallest HSV's from $A_{b}$. Let $\tilde{A}_{m}$ be the image (in modal coordinates) of $\tilde{A}_{b}$. $\tilde{A}_{m}$ is obtained by first truncating the last $k$ rows 
of $T$ and the last $k$ columns of $T^{-1}$ to obtain $\tilde{T}$ and $\tilde{T}^{+}$respectively:

$$
\begin{gathered}
\tilde{T}=\left[\begin{array}{c}
\vec{u}_{1}^{\top} \\
\vdots \\
\vec{u}_{n-k}^{\top}
\end{array}\right] \\
\tilde{T}^{+}=\left[\begin{array}{lll}
\vec{v}_{1} & \ldots & \vec{v}_{n-k}
\end{array}\right]
\end{gathered}
$$

and then computing the projection:

$$
\tilde{A}_{m}=\tilde{T}^{+} \tilde{A}_{b} \tilde{T}
$$

Continuing, we will define the total approximation error associated with $\tilde{A}_{m}$ to be the Frobenius norm of the difference of $A_{m}$ and $\tilde{A}_{m}$, so that the total percent error, $P_{t o t}$, is:

$$
P_{t o t}=\frac{\left\|A_{m}-\tilde{A}_{m}\right\|_{F}}{\left\|A_{m}\right\|_{F}} \times 100 \%
$$

where \|\|$_{F}$ is the Frobenius norm. We will then define the error with respect to the $j$ th mode of $A_{m}$ as the Frobenius norm of the difference between the $j$ th two-by-two block on the diagonal of $A_{m}$ and the corresponding two-by-two block of $\tilde{A}_{m}$. Note that by this definition, the total approximation error cannot be obtained by summing the approximation errors of the modes individually, since $\tilde{A}_{m}$ is not guaranteed to be block-diagonal; the sum of the individual errors can be less than the total error since there is a potential for non-zero entries to occur in the off-block-diagonal rows and columns. However, the error due to these offblock-diagonal terms is shared amongst multiple modes, and we decide to omit it - doing so also reduces the computational burden of the approach.

We can compute the two-by-two block, $\tilde{A}_{m_{j}}$ of $\tilde{A}_{m}$, corresponding to the twoby-two block $A_{m_{j}}$ of the $j$ th mode on the diagonal of $A_{m}$ by viewing $\tilde{T}^{+}$and $\tilde{T}$ 
as block matrices of row and column vectors respectively:

$$
\begin{gathered}
\tilde{T}^{+}=\left[\begin{array}{c}
\vec{p}_{1}^{\top} \\
\vdots \\
\vec{p}_{n}^{\top}
\end{array}\right] \\
\tilde{T}=\left[\begin{array}{lll}
\vec{q}_{1} & \ldots & \vec{q}_{n}
\end{array}\right]
\end{gathered}
$$

where $\vec{p}_{i}^{\top}$ is the $i$ th row of $\tilde{T}^{+}$and $\vec{p}_{i}$ is the $i$ th column of $\tilde{T} . \tilde{A}_{m_{j}}$ is computed by a projection using only the $(2 j)$ th and $(2 j-1)$ th rows and columns of $\tilde{T}^{+}$and $\tilde{T}$ respectively, as follows:

$$
\tilde{A}_{m_{j}}=\left[\begin{array}{c}
\vec{p}_{2 j-1}^{\top} \\
\vec{p}_{2 j}^{\top}
\end{array}\right] \tilde{A}_{b}\left[\begin{array}{ll}
\vec{q}_{2 j-1} & \vec{q}_{2 j}
\end{array}\right] .
$$

The percent error associated with the $j$ th mode is then:

$$
P_{j}=\frac{\left\|A_{m_{j}}-\tilde{A}_{m_{j}}\right\|_{F}}{\left\|A_{m_{j}}\right\|_{F}} \times 100 \% .
$$

When the percent error associated with the $j$ th mode (after $k$ states have been truncated from the model) is computed over all possible $j$ and $k$, we can obtain a table of percent error data with $k$ columns and $j$ rows. Although this data is discrete, for visualization purposes it can be plotted as a contour map in MATLAB [15] with the contour() function, as in figure 3.2 which uses the eight-mode model whose HSV's are displayed in figure 3.1.

The percent error contour plot is a key finding in this work - it provides a way of visualizing the relationship between the states of a balanced representation and the modes of a system. It is also structured so that it serves as both a frequency-response type plot (by virtue of associating each mode with its natural frequency) and as a predictive plot of which modes should be well-represented in a reduced-order model obtained by via balanced truncation. In the next chapter 


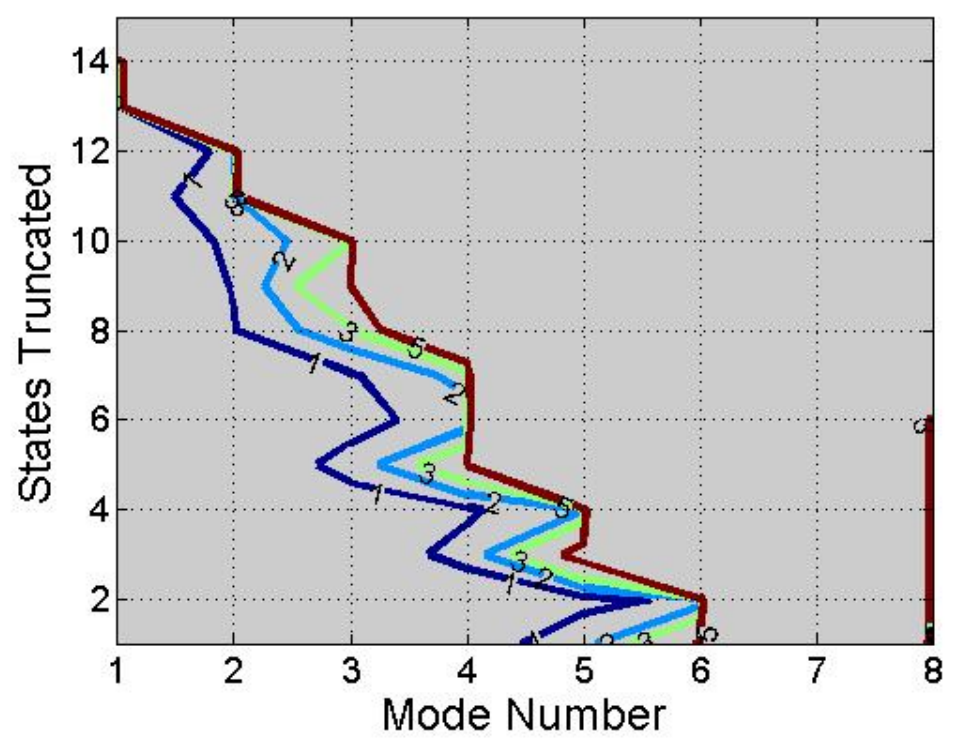

Figure 3.2: Percent error contour plot for an 8-mode model. Contours for the 1, 2, 3, and 5 percent error levels are plotted for each of the 8 modes as the number of balanced states truncated from the model is varied.

the percent error contour plot will be employed in the investigation of finite element models of an illustrative system, in conjunction with a parametric study. Balanced truncation will be applied to generate reduced-order reference models for use in an adaptive control law, and the performance of the closed-loop system observed. 


\section{Chapter 4}

\section{Parametric Studies of Illustrative Systems}

\subsection{Control Law}

The adaptive control law used for demonstrating the dynamic performance of reduced-order reference models in this work was Direct Model Reference Adaptive Control (DMRAC). Since the aim of this work is to focus on model reduction techniques rather than control algorithms, the fundamental derivations regarding the convergence and stability of the DMRAC method will be omitted, but can be found in Mehiel's dissertation [16]. As its name indicates, DMRAC belongs to a class of adaptive control laws known as direct control methods (as opposed to indirect control). DMRAC uses a set of adaptive gains to modify the behavior of the plant so that it tracks a predetermined mathematical model called the reference model. For example, the plant could be an optical telescope, and the reference model a reduced-order finite element model representative of its struc- 
ture. Note that indirect control methods differ from direct control methods in that indirect control instead focuses on system identification - an indirect control law seeks to continually refine and improve a model in the loop so that it comes to closely approximate the plant.

When a physical system's dynamics are either not fully known, or not wellrepresented by available mathematical models, adaptive control laws can be quite useful - they can accommodate a high degree of modeling error between the plant and an approximate model, unlike other approaches such as state-feedback. This makes DMRAC in particular very useful for our purposes, since we can use it to test a variety of reference models. A reference model that is a close fit to the plant will minimize the amount of controller effort that DMRAC must put forth, however, since less gain will be required to force the plant to conform to the model's behavior. This aspect of DMRAC serves as motivation to obtain accurate models of the plant.

When there is a desire to change the plant's dynamics (such as in the usual control design case - to reach certain performance objectives), a reference model with dynamics that differ from that of the plant can be utilized. To minimize the controller effort in such a case, it again makes sense to start with an accurate model of the plant. The model can then be precisely altered to change only the dynamics of the plant that need tuning, while still conforming to the plant's dynamics as closely as possible. From this perspective, an accurate reduced-order model of the plant can be desirable since its dynamics are ideally boiled down to those fundamental to the plant. In principle such a model is easy to tune, and allows a designer to efficiently modify the plant's behavior while keeping the amount of controller effort required low.

The DMRAC algorithm is applied to a system in a first-order state space 
form, with an optional disturbance term added:

$$
\begin{array}{r}
\dot{\vec{x}}_{p}=A_{p} \vec{x}_{p}+B_{p} \vec{u}_{p}+\Gamma \vec{u}_{d} \\
\vec{y}=C_{p} \vec{x}_{p}
\end{array}
$$

where $\Gamma$ is the input matrix for the disturbance vector $\vec{u}_{d}$, each entry of which is a (bounded) disturbance function. DMRAC uses a reference model in first-order state space form, so subscripts have been introduced to distinguish between the matrices and dynamical states of the plant and reference model when necessary. The reference model has the following form:

$$
\begin{array}{r}
\dot{\vec{x}}_{m}=A_{m} \vec{x}_{m}+B_{m} \vec{u} \\
\vec{y}_{m}=C_{m} \vec{x}_{m} .
\end{array}
$$

Note that the plant (4.1) produces the system output $\vec{y}$, while the reference model (4.2) accepts the control input $\vec{u}$.

A central quantity of the DMRAC algorithm is the output error, $\vec{e}_{y}$, which is defined as the difference of the outputs of the plant and reference model:

$$
\vec{e}_{y}=\vec{y}-\vec{y}_{m}
$$

DMRAC utilizes four adaptive gains: the error, input, state, and disturbance gains. In Mehiel's notation these are: $G_{e}, S_{22}, S_{21}$, and $H_{d}$ respectively. The control law for DMRAC is given in [16] as:

$$
\vec{u}_{p}=G_{e} \vec{e}_{y}+S_{21} \vec{x}_{m}+S_{22} \vec{u}_{m}+H_{d} \vec{u}_{d}
$$

The gains evolve over time according to the following adaptive gain laws:

$$
\begin{gathered}
\dot{S}_{12}=-\vec{e}_{y} \vec{x}_{m}^{\top} H_{1} \\
\dot{S}_{22}=-\vec{e}_{y} \vec{u}^{\top} H_{2} \\
\dot{G}_{e}=-\vec{e}_{y} \vec{e}_{y}^{\top} H_{3} \\
\dot{H}_{d}=-\vec{e}_{y} \vec{u}_{d}^{\top} H_{4}
\end{gathered}
$$


where $H_{1}, H_{2}, H_{3}$ and $H_{4}$ are referred to as the adaptive parameters, and are positive definite matrices of the appropriate sizes (or alternatively, non-negative real constants). The structure of the DMRAC algorithm is depicted in figure 4.1 as a control diagram, reproduced from Mehiel's disseration [16]. In our work, we will

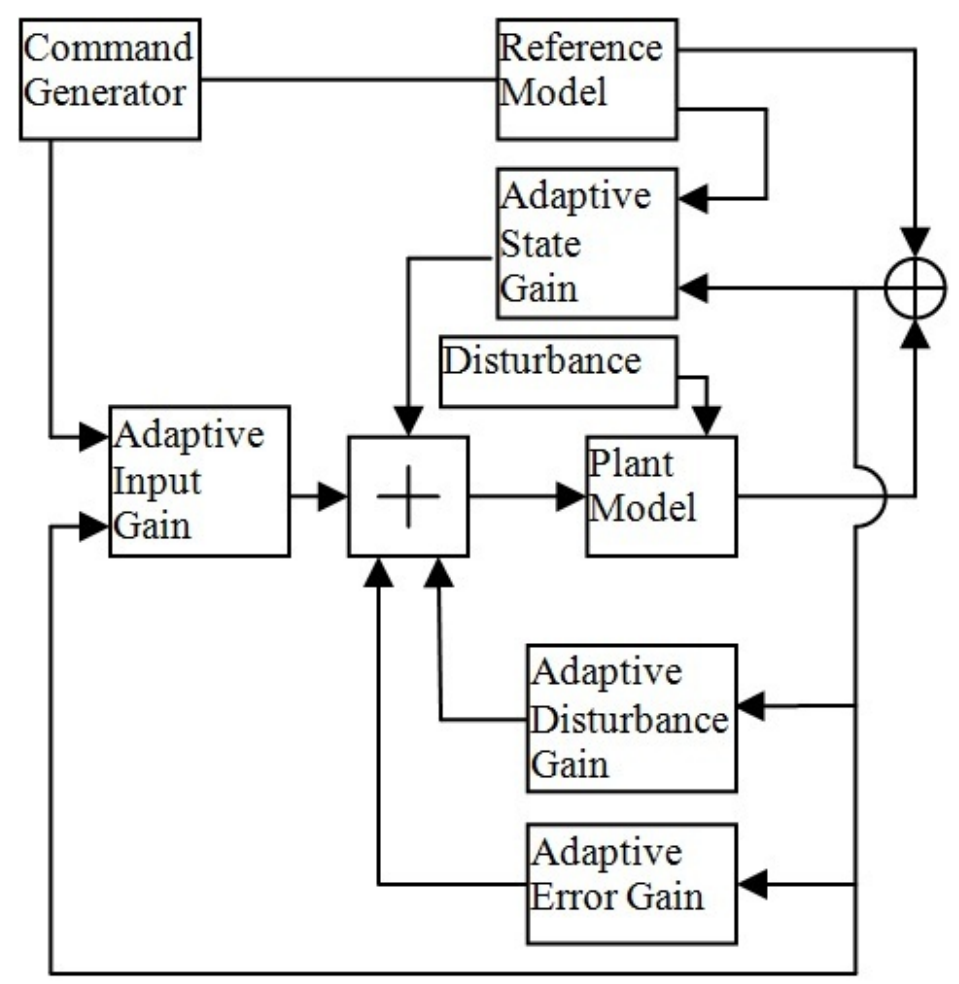

Figure 4.1: Control diagram for DMRAC.

eliminate the use of the adaptive state gain because it depends on the coordinate system of the reference model - that is, two equivalent reference models will produce different dynamics in the DMRAC algorithm. This would be unacceptable in comparing the performance of reference models since some performance gains and losses could be due solely to the choice of coordinates. We will also not make use of the adaptive disturbance gain, since this adapts to reject disturbances fed into the plant without considering the choice of reference model. In the existing DMRAC structure this can be accomplished by setting the $H_{1}$ and $H_{4}$ adaptive 
parameters to zero.

One additional nuance of the DMRAC algorithm is that proofs of stability and convergence as in [16] require that the plant satisfy a certain condition namely that it be strictly positive real (SPR). Note that while the SPR condition is required in some proofs of convergence and stability, it is not a statement that DMRAC will absolutely not work if the plant is not SPR. Following Mehiel, we will define a system $(A, B, C)$ to be SPR if it is controllable (i.e. its controllability gramian is full-rank) and there exist square matrices $P$ and $Q$ so that it satisfies the Kalman-Yacubovic conditions:

$$
\begin{array}{r}
A^{\top} P+P A=-Q^{\top} Q-L \\
P B=C^{\top}
\end{array}
$$

where $P$ is symmetric and positive definite, and $L$ is positive definite.

\subsection{Modeling an Illustrative System}

Many, if not most, vibration control problems in space structures are in some way related to optical devices due to the extreme motion-sensitivity of such systems. Examples include the deployable optical telescope that Mehiel [16] was concerned with controlling, and the optical communications link design presented by Adolph et al. [1] amongst many others. We will consider the problem of controlling an optical mirror segment. To simplify the analysis of this illustrative system, we will assume the mirror can be modeled as a cantilever beam of constant

rectangular cross section. The mirror segment will be made out of beryllium, and be one hundred millimeters long, ten millimeters wide, and one millimeter thick. The mirror segment will be both sensed and actuated by a linear electric motor, modeled as providing a point force applied to the center of the tip of the can- 
tilever. It is assumed that the velocity of the cantilever tip can be measured by the back electromotive force (back-emf) of the motor. A sketch of the model is presented in figure 4.2. The damping ratio was assumed to be 0.09 for all modes.

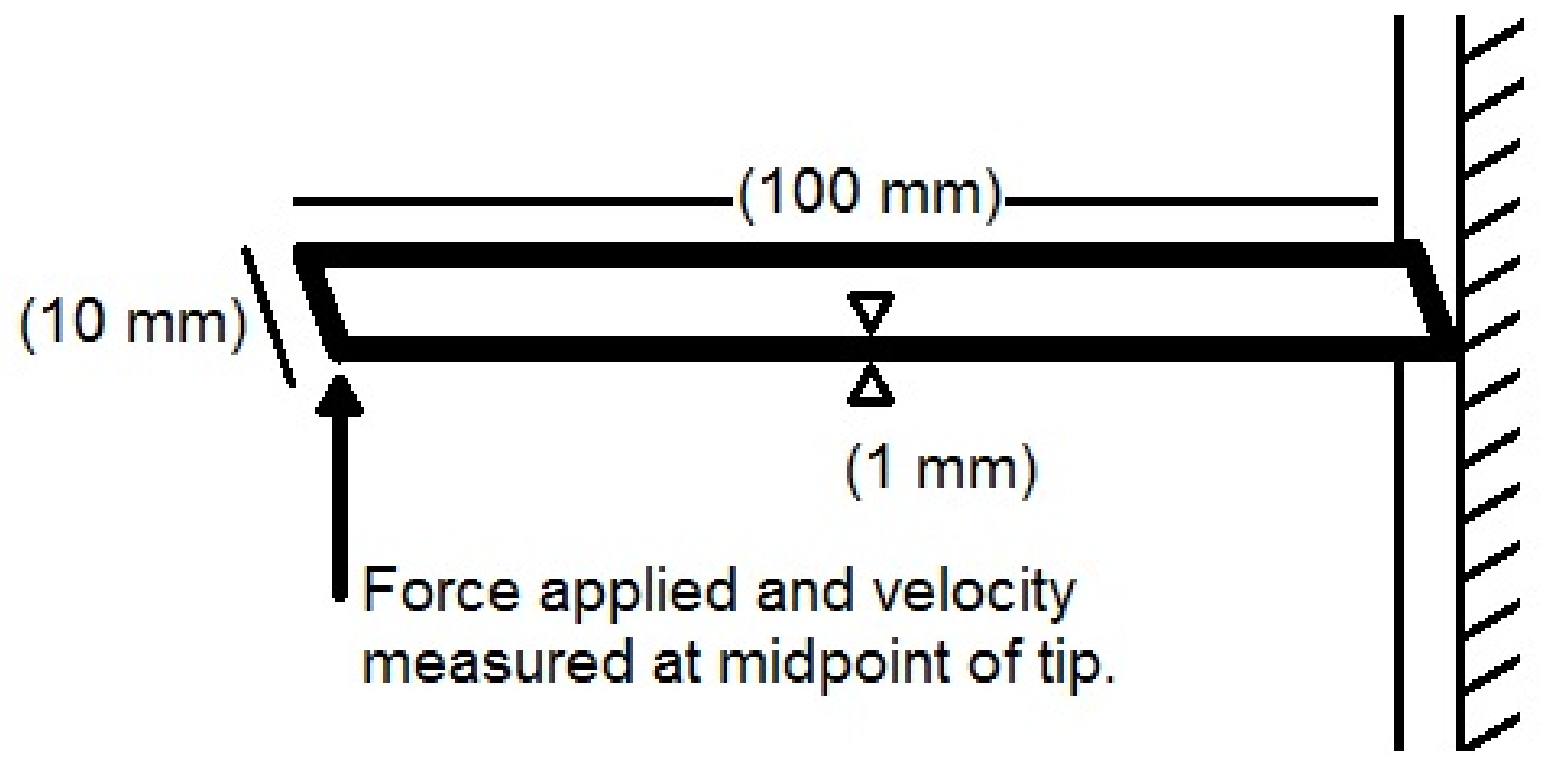

Figure 4.2: Diagram of a beryllium mirror segment.

The boundary conditions (which are due to the built-in end) were enforced by truncating the rows and columns corresponding to that endpoint node.

\subsection{Investigation of Illustrative System}

In beginning our investigation of the finite element models of the cantilevered mirror segment structure, we will first consider how a contour map of the percent error with respect to its various modes (as in figure 3.2) changes as the discretization of the model is made coarser or finer. Finite element models of the mirror segment were constructed using five, ten, fifteen, and twenty EulerBernoulli beam elements respectively. Contour maps for these four finite element models of the structure are displayed in the figure 4.3. In these contour maps, 
the one, two, three, and five percent error contour levels have been plotted. For comparison, their log-scale plots of Hankel singular values are displayed in figure 4.4 .
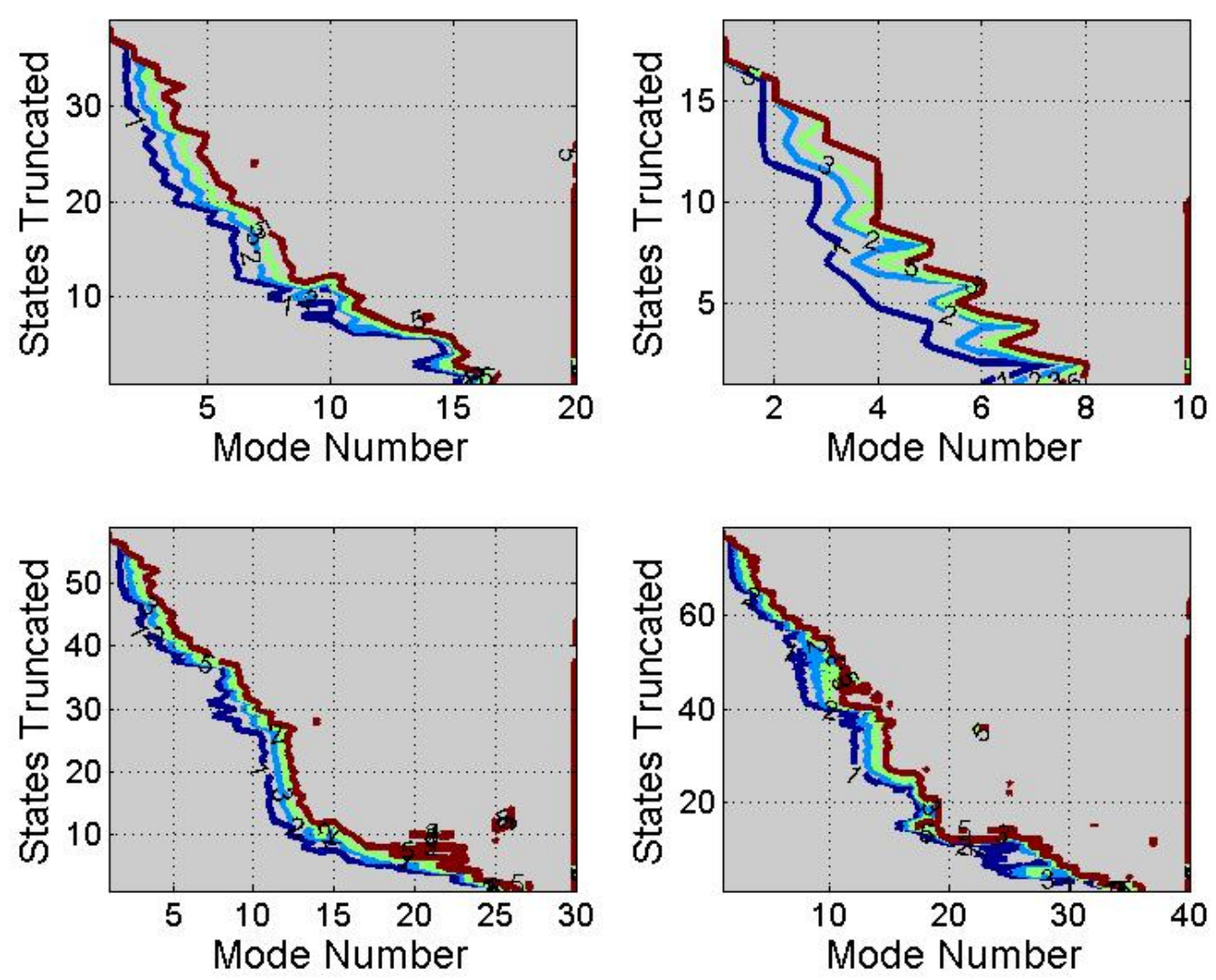

Figure 4.3: Percent error contour plots of beam models. Counterclockwise from the top right: the percent error contour plots of the 5, 10, 15, and 20 element models of the mirror segment.

Some interesting patterns are revealed in the percent error contour figures. Firstly, notice that (particularly in the fifteen and twenty element cases) a kink occurs after which the slope of the percent error contour becomes relatively shallow for the highest-order modes. Apparently the modes can be separated into a group of low-order modes that are persistent as states are truncated, and another 

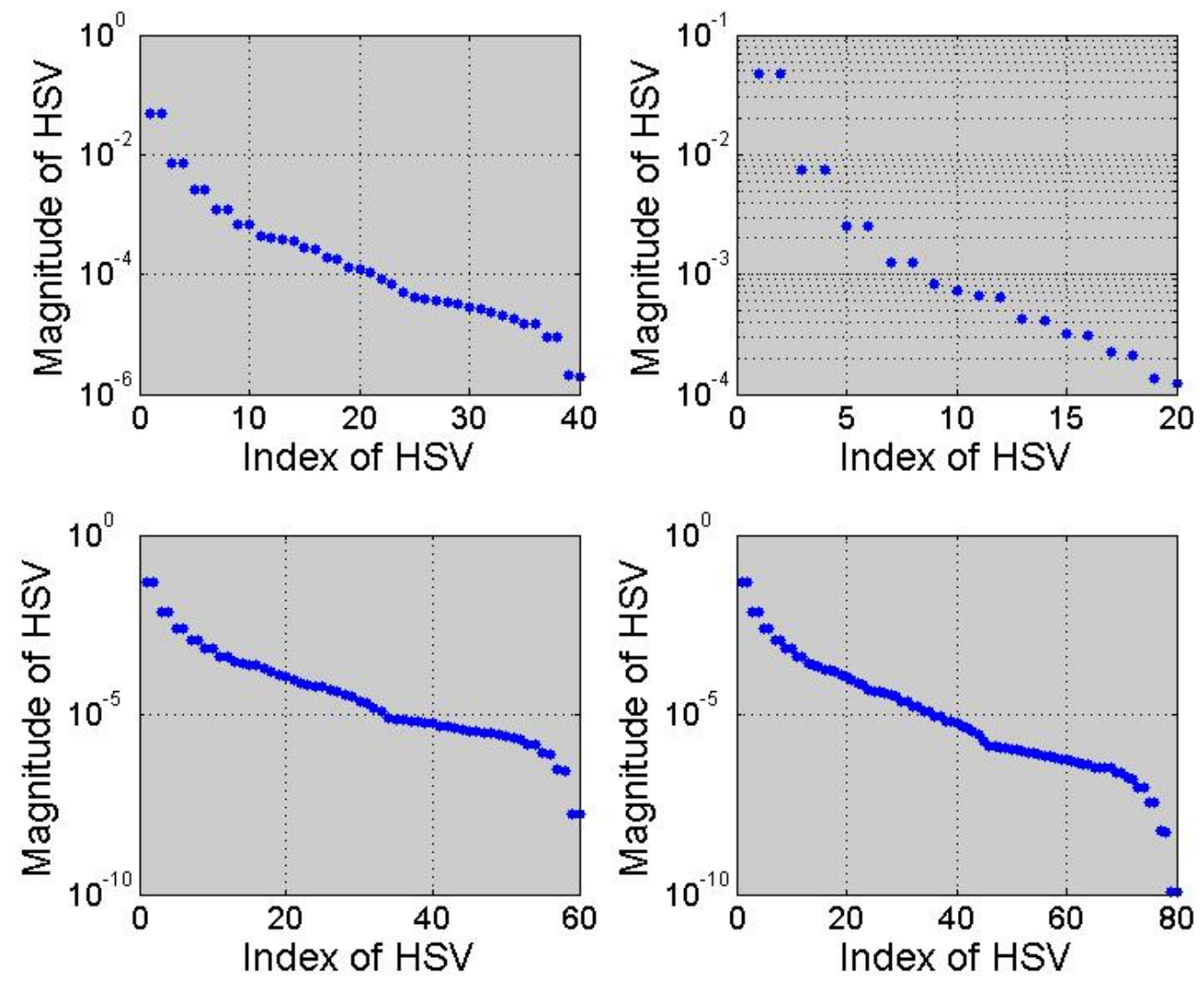

Figure 4.4: Log-scale HSV lots of beam models. Counterclockwise from the top right: log-scale plots of HSV's for the 5, 10, 15, and 20 element models of the mirror segment.

group of high-order modes that vanish quickly from the model. This seems to suggest that the low-order modes are among the most physically meaningful ones that the model captured, with the rest potentially being computational artifacts. The great degree of interrelation between the high-order modes with respect to the states of the balanced coordinate system suggests that they all have similarly low measures of controllability and observability.

Also present in the percent error contours is a quirk that shows up in all of the models; the highest-order is very highly ranked in terms of its control- 
lability/observability. This is not a peculiarity of the contour map method or the balancing algorithm, but of the finite element model itself - the same phenomenon is very pronounced in the Bode plots (figure 4.5) of the four systems, produced by MATLAB's [15] bode() function. A closer inspection of the Bode
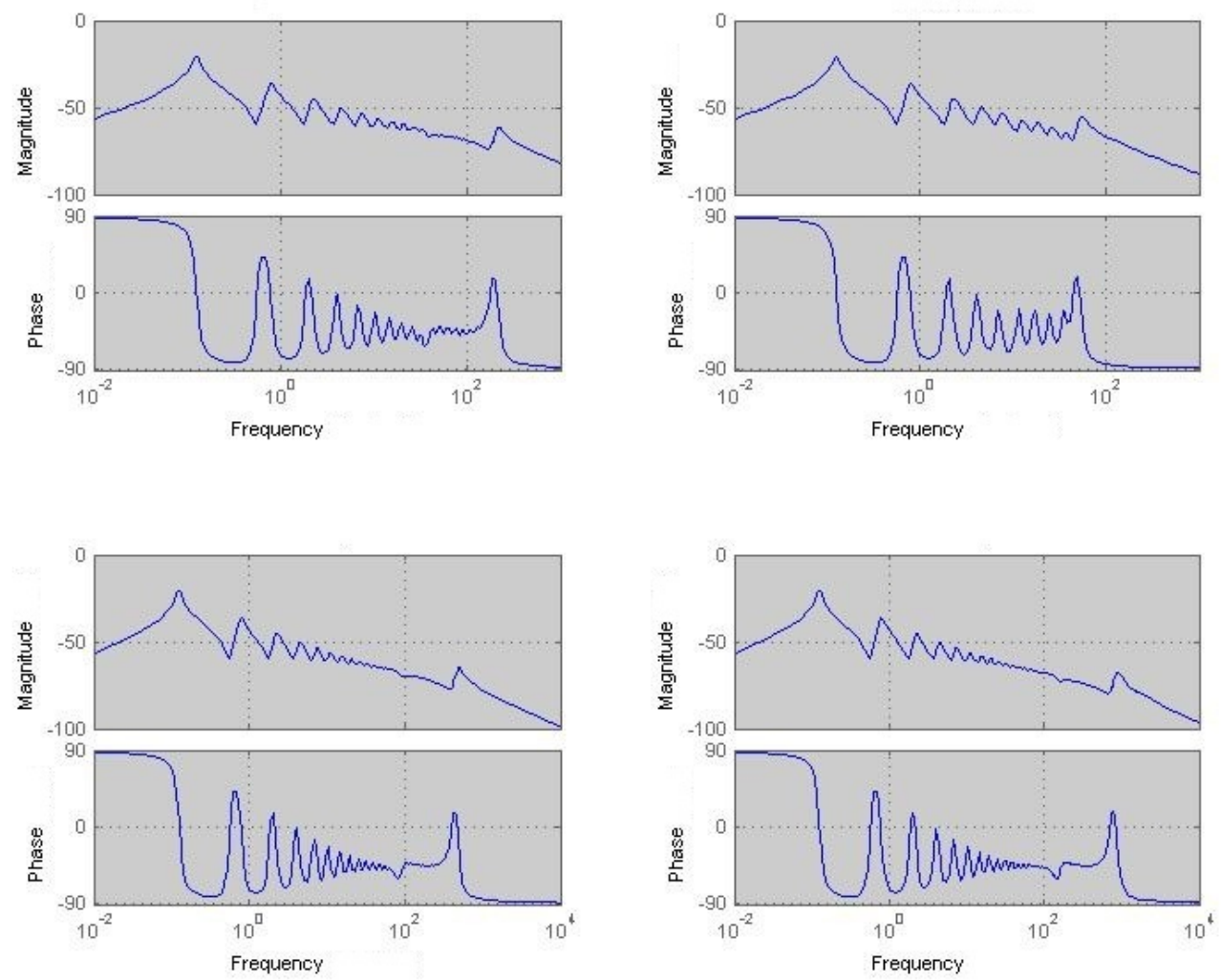

Figure 4.5: Bode plots of beam models. Counterclockwise from the top right: the Bode plots of the $5,10,15$, and 20 element beam models. Units are in $\mathrm{dB}$, degrees, and radians per $10^{-4}$ seconds.

plots of the systems shows that they appear to represent the behavior one might expect from a cantilever beam, until the frequency exceeds a certain limit in each case. Adding more elements to the model is seen to increase this limit, extending the range of frequencies with realistic behavior. This coincides with our 
physical intuition, since adding more elements allows the beam model to deflect into higher-frequency mode-shapes. In the ten-element model realistic behavior is lost at frequencies higher than about $3.2 \times 10^{5}$ radians per second $\left(3.2 \times 10^{1}\right.$ on the Bode plot scale), whereas in the twenty-element model the limit is nearly an order of magnitude higher at $1.4 \times 10^{6}$ radians per second. Observing this, it appears that a preliminary modal truncation of the highest-frequency modes of a model can sometimes be beneficial from a modeling standpoint. Such modes are unlikely to be physically meaningful, and when one or more of them also have unrealistically high gain they can introduce error to the model and complicate the process of computing a reduced-order balanced model. Other model reduction approaches that account for sensors and actuators may be similarly affected.

Further inspection of Bode plots (with the models altered to incorporate a near-zero damping ratio to sharpen peaks due to resonance) reveals that a discernible gap in resonant frequency exists between exactly the first half of the modes and the second half in all four models. Figure 4.6 shows the Bode plot for the 20-element model, with damping ratio reduced to $1 \times 10^{-12}$ for the purposes of visualizing its resonant frequencies. The phenomenon exemplified in figure 4.6 suggests that our finite element model construction method consistently produces dynamical behavior corresponding to what we would expect from a physical cantilever beam in exactly the first half of its modes of vibration, with the rest of the modes being computational artifacts. Reviewing figure 4.3, notice that as the number of elements in the model is increased, the plots come to reflect this phenomenon more closely - a vertical line separating the first half of the modes from the second half would intersect the plotted percent error contours very near the point at which the slope of the contours flattens in the fifteen and twenty element cases. 


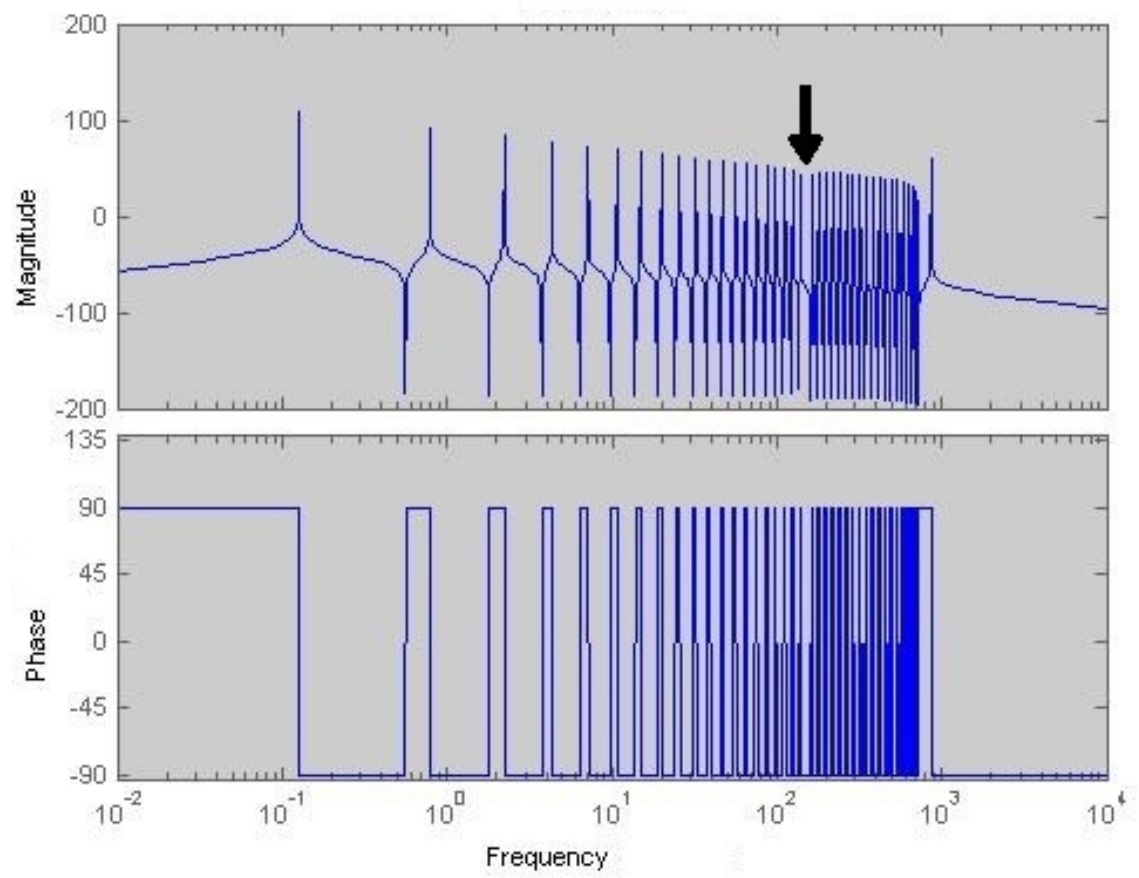

Figure 4.6: Bode plot with gap. The arrow indicates a gap in frequency between the first and last 20 modes of the model.

Examining the log-scale plots of the HSV's in figure 4.4, we see that a similar flattening occurs somewhat after the halfway point along the HSV index, suggesting that this may be a good place to truncate the model (under the HSV plot method). This reaffirms that that the model's states can be partitioned into high-gain and low-gain classes of roughly equal size. Unlike the Bode diagrams and percent error contour plots, however, frequency data is missing from the HSV plot approach. As such, the HSV's corresponding to the balanced states of the erroneous high-order mode are indistinguishable from other high-gain balanced states.

Continuing forward, we will select the fifteen-element model as our system for further study. Enough elements were included in this model for the percent error 
contour plot to be clearly defined relative to the other cases. Considering the plot it appears that truncating thirty-six states is a good option - below this point the slope of the percent error contour is quite steep, indicating that these states can be truncated without greatly effecting the modal characteristics of the model. The truncation point is shown by the black line in figure 4.7. Note however, that this truncation point still includes effects due to the high-gain high-order modes, in particular, the highest-order mode. We are also not completely sure at this point whether truncating even further would be an acceptable proposition. Motivated by our desire to eliminate the high-order mode, we will also take a slightly different approach.

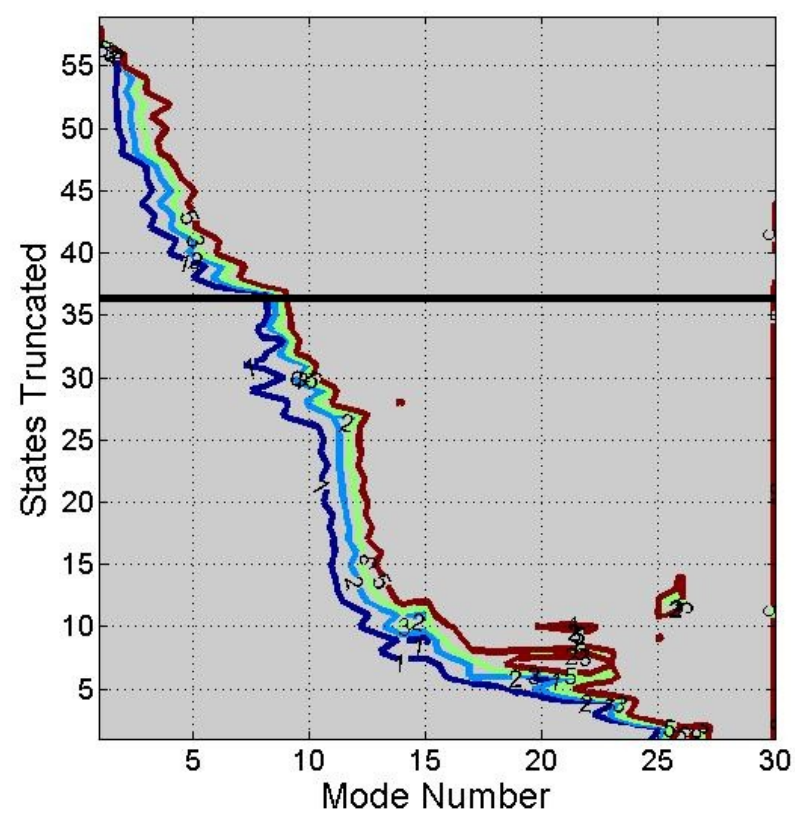

Figure 4.7: Percent error contour plot with truncation line. The truncation point for the 15-element model was chosen using the geometry of the percent error contour plot, as indicated by the black horizontal line.

The contour plot is not the only way to make use of the percent error data - 
another plot can be constructed to help identify instances where the truncation of balanced states in the usual order results in the loss of structural modes in an order different than one would expect from their mode numbers (with highorder modes being truncated first). Since we know a state-transition matrix with $(2 n+1)$ states can have at most $n$ conjugate pairs of eigenvalues, it follows that if we can truncate $k$ states to a point where the mean percent error of the first $\lfloor(n-k) / 2\rfloor$ modes is small, we will have successfully eliminated the contributions of the high-gain high-order modes from the model. Conversely, if as we truncate balanced states the mean percent error associated with the first $\lfloor(n-k) / 2\rfloor$ modes grows, then we are either imparting large amounts of error to multiple modes through the truncation of only a couple states, or we are effectively truncating modes in an order that does not respect their mode numbers. A plot of the mean percent error levels is shown in figure 4.8.

Like the percent error contour plot, the mean percent error plot also gives us an idea of how well the modes are represented in the balanced truncated model. In the plot we notice that as we truncate though the last few modes, the mean percent error steadily decreases until it reaches its smallest value when it becomes the error with respect to the fundamental mode alone. We are effectively seeing that as we truncate through the lowest-order modes, the balanced truncation is behaving more and more like a modal truncation, as one might expect - the lowest order modes clearly dominate the dynamics of this system.

Observing figure 4.8 , we conclude that truncating the first fifty states of the model is a reasonable proposition - it is at this point that the mean percent error first becomes less than any of its previous values. We have also eliminated the contributions of the high-gain, high-order modes from the model, but at the cost of additional modeling error due to the truncation of more states. In the 


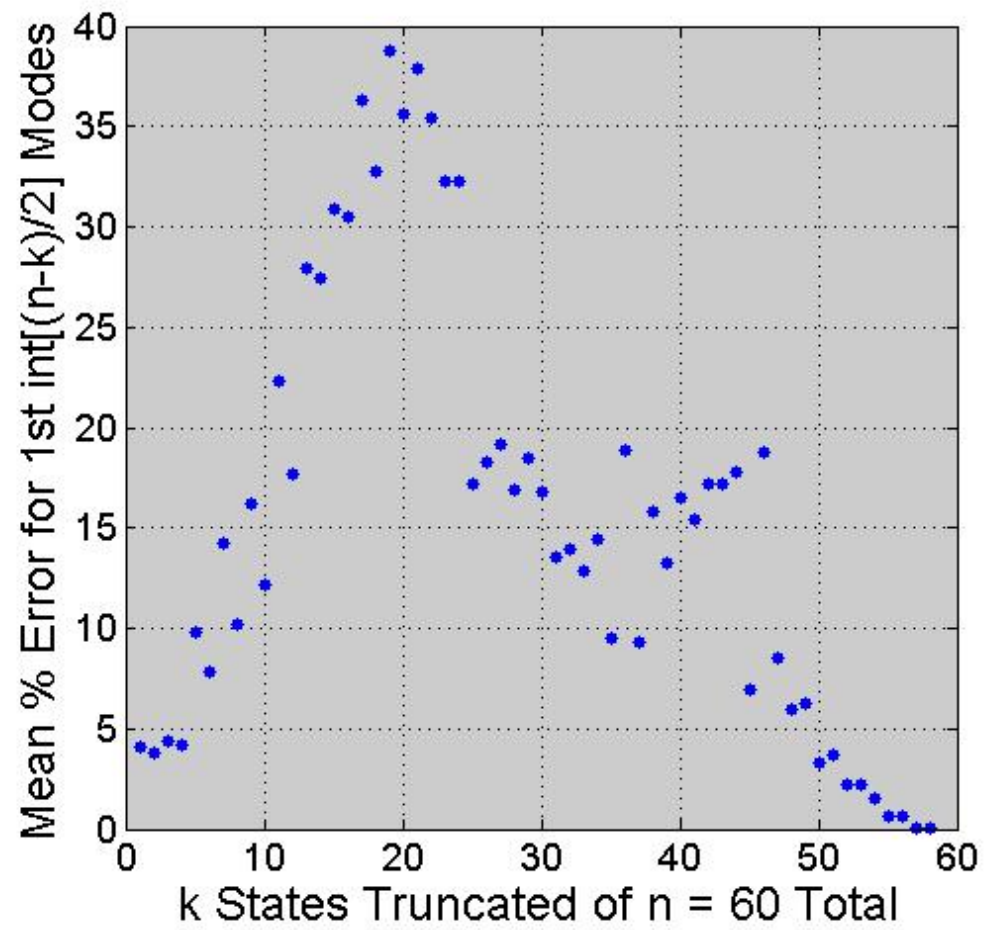

Figure 4.8: Plot of mean percent error versus states truncated. The plot depicts the mean percent error for the first $\lfloor(n-k) / 2\rfloor$ modes after $k$ states have been truncated from the 15-element model.

next section we will investigate the dynamic behavior of a (DMRAC) controlled system using both the twenty-four state and ten-state balanced truncated models as reference models.

\subsection{Parametric Study}

If the mirror segment in the illustrative system is commanded to deflect to a new position, the force input excites structural modes and causes vibration. In this study we will implement DMRAC on the illustrative system and then modify the reference model to change the dynamic response, with the goal of attenuating vibration and decreasing the settling time. The plant in the DMRAC algorithm 
was chosen to be the fifteen-element model of the cantilevered mirror segment, with the last fifteen modes truncated via modal truncation - these modes are almost undoubtedly computational artifacts and would not correspond to the dynamics of a physical system, which the plant would be in practice.

The full-order finite element model and the twenty-four state and ten-state balanced truncated models were used as reference models to observe the resulting performance. To achieve a faster settling time, a second ten-state model was produced with altered modal damping. This was accomplished by transforming the model to the usual modal form via the method discussed in chapter 2 . The first and second modes of this model then had their damping ratios increased to 0.5 from their original values.

The governing equations for DMRAC, (4.1) through (4.5), were implemented in a MATLAB script and were numerically integrated using the default settings on MATLAB's ode45() function. To ease the numerical integration process, the system units were carefully scaled. The mass, length and time units used in the computation were $10^{-6}$ kilograms, $10^{-2}$ meters, and $10^{-4}$ seconds respectively. A ten Newton step input was used as the input command, and the adaptive parameters for the adaptive error and adaptive input gains were both set to a value of one hundred.

The simulations computed the system response over a duration of 0.05 seconds in each case, with the ode45() function instructed to return the integration values at $2^{16}$ evenly spaced time steps for plotting purposes. In particular, the number of points was chosen to be a power of two for the generation of amplitude spectrum plots. Plots of the output signals of the controlled system and its reference model are presented in figure 4.9 for each of the four cases, and figure 4.10 displays the corresponding output errors. 

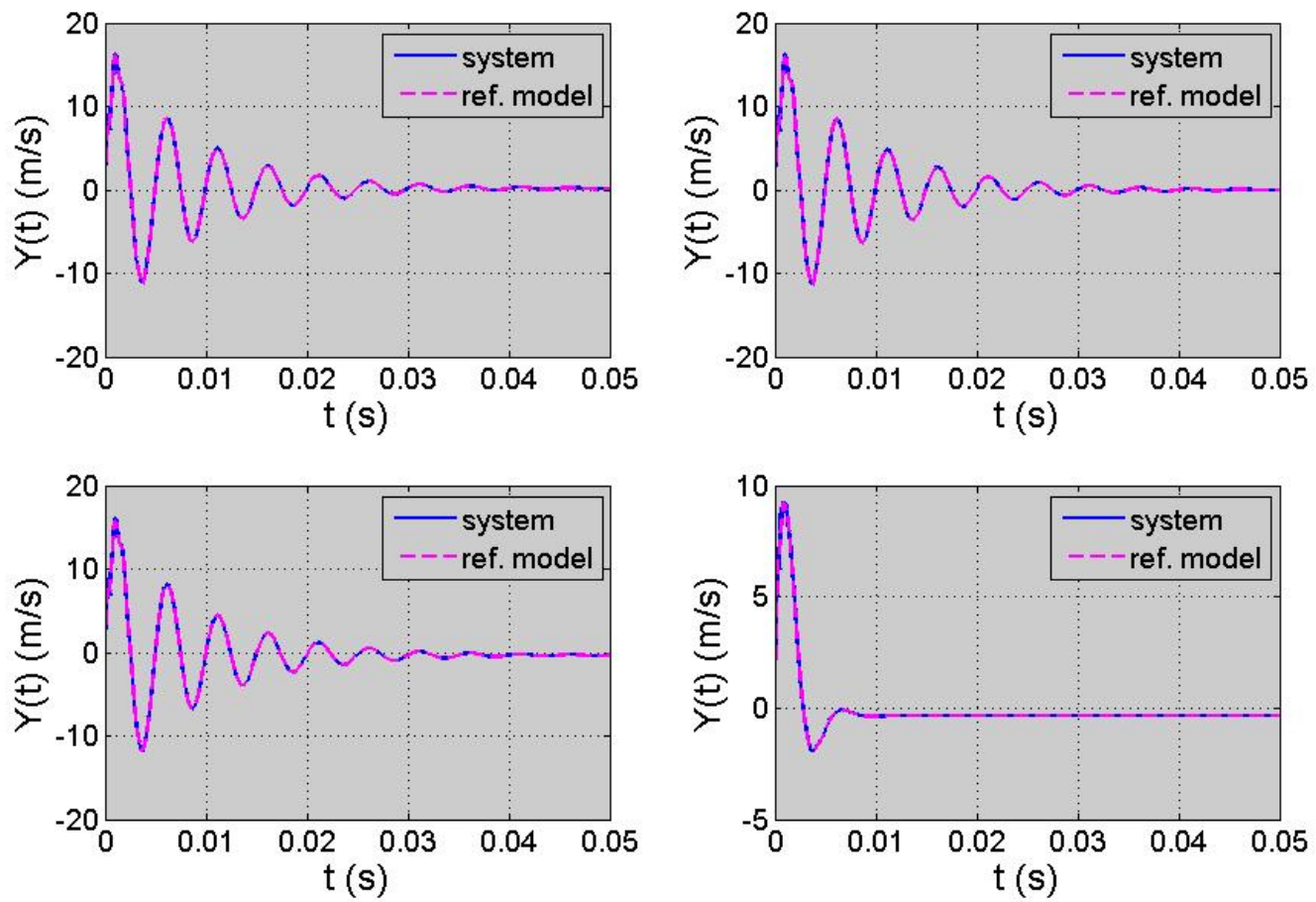

Figure 4.9: Output signals of controlled systems, with 15-mode plant. Counterclockwise from the top right: output signals of the controlled system and its reference model, using full-order, 24-state, and 10-state reference models.

As we can see in figure 4.9, the artificially-damped reference model produced the most noticeable effect on the controlled system - the increase in damping considerably reduced the vibration and maximum velocity attained. The differences between the other three cases are too small to be visually distinctive.

Regarding the plots of the output errors in figure 4.10, not much can be gained from a glance, except that the settling time for the fourth case with increased damping is slightly longer than the others. This follows our expectations, since the heavily-damped model presents a significant departure from the dynamics of the plant that DMRAC must accommodate for. Regardless, the settling times for the output error are visibly much smaller than the settling times for the system 
output - DMRAC successfully conforms the behavior of the plant to match that of the reference models long before the model's vibrations settle.
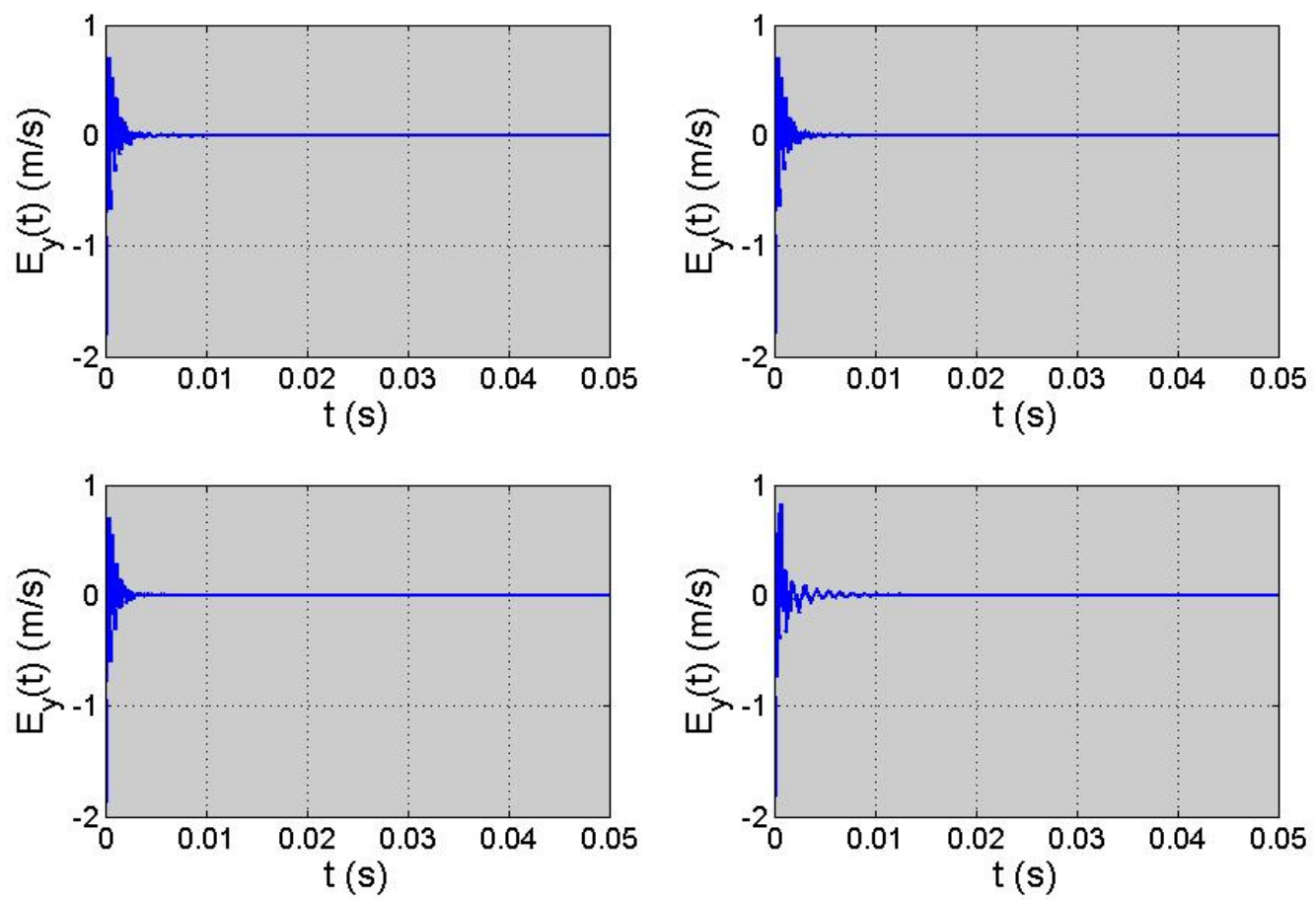

Figure 4.10: Output error signals of controlled systems, with 15-mode plant. Counterclockwise from the top right: output error signals of the controlled system and its reference model, using full-order, 24-state, and 10-state reference models.

Table 4.1 summarizes some of the dynamic behavior of the systems, and includes the five percent settling times, maximum and minimum values, and final values of the four cases. Note that since the system output is a velocity signal and the initial plant velocity is zero, the settling time is the time it takes for the system to return to within five percent of zero velocity.

One effect immediately noticeable in table 4.1 is what appears to be an increase in steady-state error as more states are truncated from the balanced reference model. Because the output signal is supposed to be the velocity of the 


\begin{tabular}{|c|c|c|c|c|c|}
\hline Signal & States & $5 \%$ Settling Time & Min. Value & Max. Value & Final Value \\
\hline$\vec{y}$ & 60 & $2.64 \mathrm{E}-02$ & $-1.14 \mathrm{E}+01$ & $1.62 \mathrm{E}+01$ & $7.05 \mathrm{E}-03$ \\
\hline & 24 & $2.67 \mathrm{E}-02$ & $-1.12 \mathrm{E}+01$ & $1.62 \mathrm{E}+01$ & $1.63 \mathrm{E}-01$ \\
\hline & 10 & $2.92 \mathrm{E}-02$ & $-1.18 \mathrm{E}+01$ & $1.60 \mathrm{E}+01$ & $-3.35 \mathrm{E}-01$ \\
\hline & 10 & $5.51 \mathrm{E}-03$ & $-1.93 \mathrm{E}+00$ & $9.29 \mathrm{E}+00$ & $-3.42 \mathrm{E}-01$ \\
\hline$\vec{y}_{m}$ & 60 & $2.65 \mathrm{E}-02$ & $-1.14 \mathrm{E}+01$ & $1.59 \mathrm{E}+01$ & $6.95 \mathrm{E}-03$ \\
\hline & 24 & $2.67 \mathrm{E}-02$ & $-1.12 \mathrm{E}+01$ & $1.60 \mathrm{E}+01$ & $1.64 \mathrm{E}-01$ \\
\hline & 10 & $2.93 \mathrm{E}-02$ & $-1.18 \mathrm{E}+01$ & $1.58 \mathrm{E}+01$ & $-3.38 \mathrm{E}-01$ \\
\hline & 10 & $5.56 \mathrm{E}-03$ & $-1.89 \mathrm{E}+00$ & $9.22 \mathrm{E}+00$ & $-3.45 \mathrm{E}-01$ \\
\hline$\vec{y}-\vec{y}_{m}$ & 60 & $1.97 \mathrm{E}-03$ & $-1.79 \mathrm{E}+00$ & $6.98 \mathrm{E}-01$ & $9.44 \mathrm{E}-05$ \\
\hline & 24 & $1.98 \mathrm{E}-03$ & $-1.79 \mathrm{E}+00$ & $7.04 \mathrm{E}-01$ & $-1.01 \mathrm{E}-03$ \\
\hline & 10 & $1.66 \mathrm{E}-03$ & $-1.86 \mathrm{E}+00$ & $7.04 \mathrm{E}-01$ & $2.46 \mathrm{E}-03$ \\
\hline & 10 & $2.57 \mathrm{E}-03$ & $-1.81 \mathrm{E}+00$ & $8.26 \mathrm{E}-01$ & $2.44 \mathrm{E}-03$ \\
\hline
\end{tabular}

Table 4.1: Performance data for controlled systems, with 15-mode plant. Units are in seconds and meters per second.

tip of a cantilever under a constant load, the signal should converge to zero as an equilibrium is reached; instead a small constant signal is observed in the output. When we consider, however, that balanced truncation does not preserve the second-order form of a finite element model, this constant signal makes sense - it is actually part of a position-related signal being fed back through the truncated sensor matrix. When the ten-state system was converted to modal form so that modal damping could be added, this effect was clearly seen in the transformed sensor matrix.

Table 4.1 also reflects the small settling times for the output error in comparison with that of the system output. Interestingly, the settling time for the unaltered ten-state model's output error is the fastest of all four models, at about 1.7 milliseconds, suggesting that this model has captured the fundamental dynamics of the plant quite well. We also see that adding damping to the model increased the setting time for the output error by about fifty percent, but in the process the settling time for the system output was decreased by about eighty 
percent.

The amplitude spectrums of the output signals of the system and its reference model were computed using MATLAB's discrete Fourier transform, fft(), to show how the choice of reference model affected the frequency response of the closedloop system. The data obtained from $\mathrm{ftt}()$ is displayed on a log-scale plot in figure 4.11 .
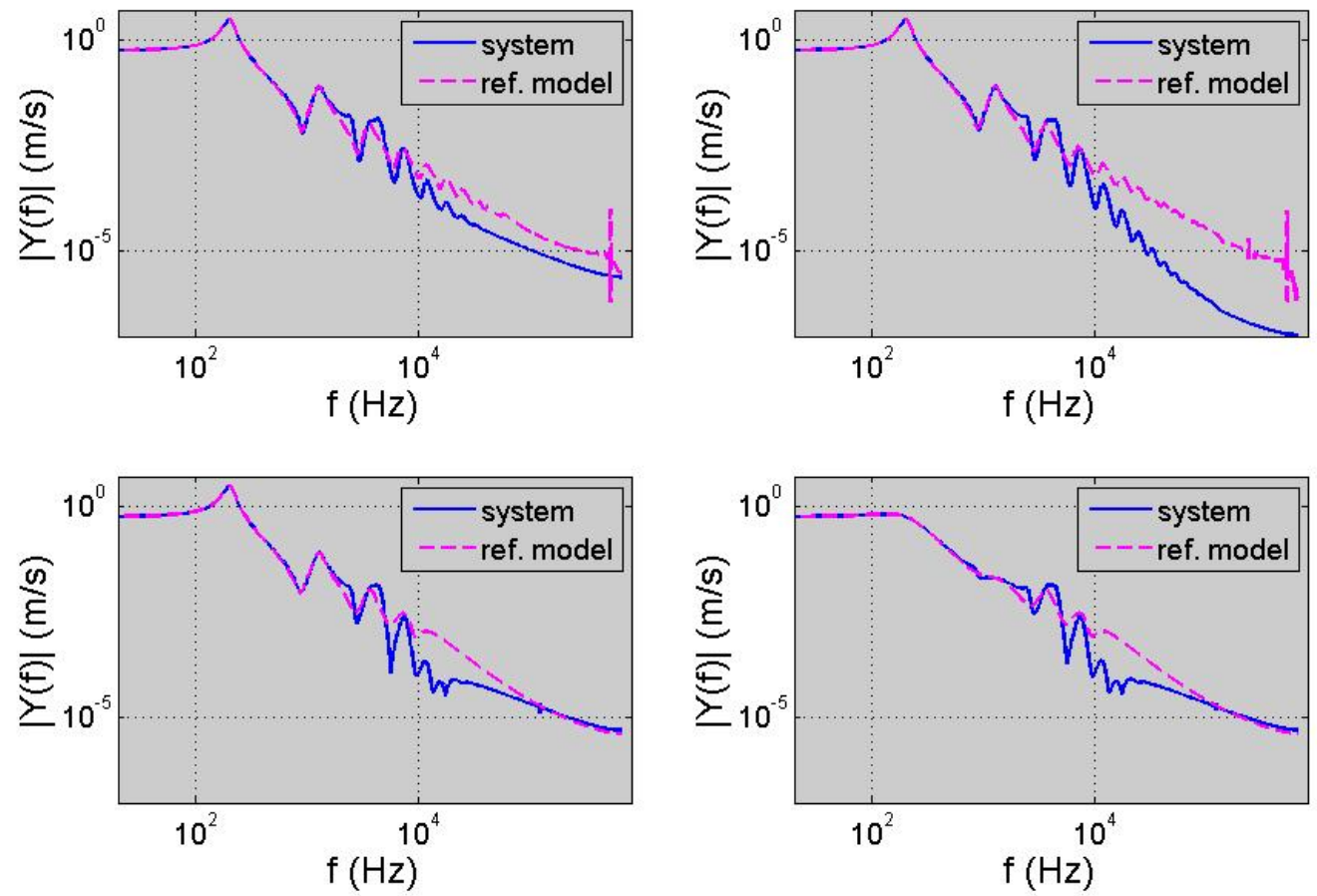

Figure 4.11: Amplitude spectrums of system outputs, with 15-mode plant. Counterclockwise from the top right: amplitude spectrums of the output signals of controlled systems using full-order, 24-state, and 10-state reference models.

Amplitude spikes in the highest frequencies of the reference model output signal are visible in the first and second cases. This is undoubtedly due to the inclusion of some erroneous high-order modes in these models, in particular the high-gain highest-degree mode of the system, as identified in the analysis of chap- 
ter 3. As predicted by the percent error contour plots, the ten-state models did not include any of these high frequency modes, as can clearly be seen in their amplitude spectra. It is interesting to note that the high-frequency behavior of the reference model was not correspondingly observed in the system output. Apparently the DMRAC algorithm could not force the plant to oscillate at such high frequencies.
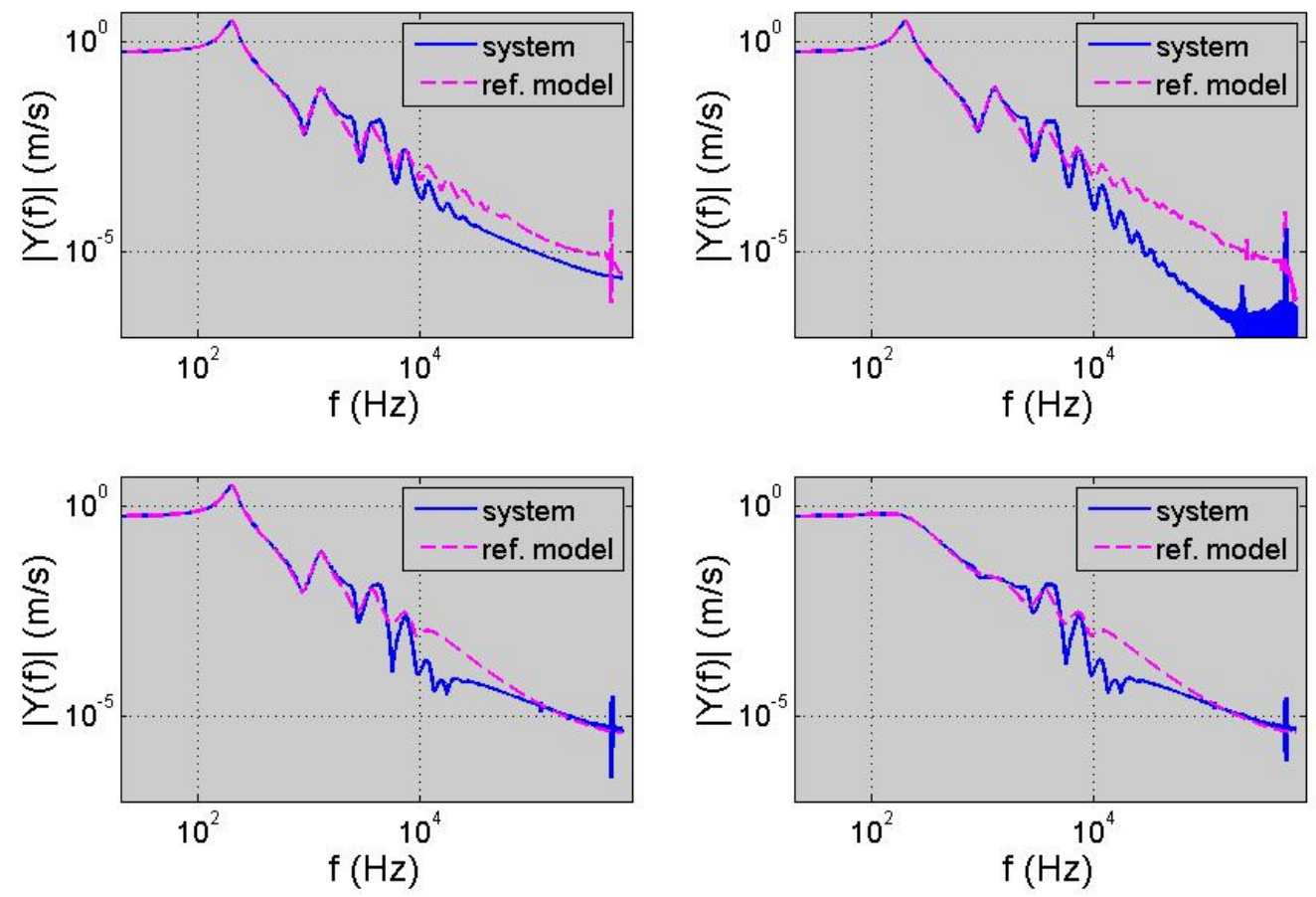

Figure 4.12: Amplitude spectrums of system outputs, with 30-mode plant. Counterclockwise from the top right: amplitude spectrums of the output signals of controlled systems using full-order, 24-state, and 10 -state reference models.

The amplitude spectrum also illustrates the effects of the increased modal damping in the frequency response of the controlled system - the response of the system at its first two resonant frequencies is attenuated as expected, with the resonances due to other modes being relatively unaffected. 
The case in which the full-order finite element model was chosen to be the plant was also considered. The resulting plots of system outputs and output errors did not vary appreciably from the cases employing the truncation of the last half of the full-order system's modes, except in the case of the amplitude spectrum plots. In figure 4.12 we see that amplitude spikes at high frequencies in the system output due to the erroneous high-order modes are now visible. The inclusion of the high-order modes in the plant model was apparently necessary before we could observe their dynamics in the system output, as can be seen in the contrast between figures 4.10 and 4.12. In the first case displayed in figure 4.12, high frequency noise due to the coupling between the plant and reference model (which were identical) is evident.

\begin{tabular}{|c|c|c|c|c|c|}
\hline Signal & States & $5 \%$ Settling Time & Min. Value & Max. Value & Final Value \\
\hline$\vec{y}$ & 60 & $2.64 \mathrm{E}-02$ & $-1.14 \mathrm{E}+01$ & $1.62 \mathrm{E}+01$ & $7.10 \mathrm{E}-03$ \\
\hline & 24 & $2.67 \mathrm{E}-02$ & $-1.12 \mathrm{E}+01$ & $1.62 \mathrm{E}+01$ & $1.63 \mathrm{E}-01$ \\
\hline & 10 & $2.92 \mathrm{E}-02$ & $-1.18 \mathrm{E}+01$ & $1.60 \mathrm{E}+01$ & $-3.35 \mathrm{E}-01$ \\
\hline & 10 & $5.51 \mathrm{E}-03$ & $-1.93 \mathrm{E}+00$ & $9.29 \mathrm{E}+00$ & $-3.42 \mathrm{E}-01$ \\
\hline$\vec{y}_{m}$ & 60 & $2.65 \mathrm{E}-02$ & $-1.14 \mathrm{E}+01$ & $1.59 \mathrm{E}+01$ & $7.05 \mathrm{E}-03$ \\
\hline & 24 & $2.67 \mathrm{E}-02$ & $-1.12 \mathrm{E}+01$ & $1.60 \mathrm{E}+01$ & $1.64 \mathrm{E}-01$ \\
\hline & 10 & $2.93 \mathrm{E}-02$ & $-1.18 \mathrm{E}+01$ & $1.58 \mathrm{E}+01$ & $-3.38 \mathrm{E}-01$ \\
\hline & 10 & $5.56 \mathrm{E}-03$ & $-1.89 \mathrm{E}+00$ & $9.22 \mathrm{E}+00$ & $-3.45 \mathrm{E}-01$ \\
\hline$\vec{y}-\vec{y}_{m}$ & 60 & $1.97 \mathrm{E}-03$ & $-1.79 \mathrm{E}+00$ & $6.98 \mathrm{E}-01$ & $5.46 \mathrm{E}-05$ \\
\hline & 24 & $1.98 \mathrm{E}-03$ & $-1.79 \mathrm{E}+00$ & $7.03 \mathrm{E}-01$ & $-1.01 \mathrm{E}-03$ \\
\hline & 10 & $1.67 \mathrm{E}-03$ & $-1.86 \mathrm{E}+00$ & $7.03 \mathrm{E}-01$ & $2.45 \mathrm{E}-03$ \\
\hline & 10 & $2.57 \mathrm{E}-03$ & $-1.81 \mathrm{E}+00$ & $8.26 \mathrm{E}-01$ & $2.50 \mathrm{E}-03$ \\
\hline
\end{tabular}

Table 4.2: Performance data for controlled systems, with 30-mode plant. Units are in seconds and meters per second.

As summarized in table 4.2 , the performance of the system was quite comparable to the earlier case in which a more realistic plant was used. The data sets in tables 4.1 and 4.2 are nearly identical, suggesting that the presence of the erroneous high-order modes in the plant turned out to have had a near-negligible 
effect on the system performance as a whole, likely due to the transient nature of the dynamics.

Reviewing the results of the simulations, overall it seems that the mean percent error plot best predicted the appropriate place to truncate the model unlike the percent error contour and HSV plots, it's output is a single quantity with a simple interpretation, as opposed to a diagram possibly subject to multiple interpretations. 


\section{Chapter 5}

\section{Conclusion}

This work produced some useful tools for visualizing how the modes of a model in first-order form can be related to its balanced states - the percent error contour and mean percent error plots. In the percent error contour plot, we also found a way to provide a designer with frequency response information that is usually absent from balanced model reduction approaches. The structure of the percent error contour plots developed in chapter 3 is such that frequency information is displayed simultaneously for the various reduced-order models that can be produced using the usual balanced truncation algorithm. When frequency information is important to the problem at hand, this can give a designer more information about where to truncate the model than simply analyzing the Hankel singular values would provide.

The mean percent error plot furthered our knowledge of how the balanced states were related to the modes, and allowed us to select a truncation point that eliminated computational artifacts from the model while capturing the fundamental dynamics of the system well. It should be noted that although in our case, simply ensuring the first few modes remained well-represented in the model 
was sufficient, other problems may feature more complicated objectives, such as representing a different subset of the modes. Similar ideas can be used in such problems, such as computing the mean percent error over a fixed subset of modes, possibly using a weighted mean.

In addition to demonstrating the effectiveness of various reference models, the simulations using DMRAC provided an opportunity to improve the system performance. After the reduced-order balanced model was converted to modal form using the methods of chapter 2 , and its modal damping increased, vibration in the system was reduced and the settling time reduced by about eighty percent.

\section{$5.1 \quad$ Future Work}

Although the focus of this work was on balanced reduced-order modeling, some limitations and drawbacks of balanced model reduction were noted in addition to its obvious computational demands. For example, a balanced truncated model is not guaranteed to retain its underlying second-order structure. Consequently the position and velocity states internal to the model can become blurred, as was observed in the output dynamics of the reduced-order models. When the balanced reduced-order model was modalized, this change manifested itself as position-measurement terms in a sensor matrix that previously sensed only velocity.

No model reduction method is without such drawbacks, but these in particular make balanced model reduction rather unappealing for use on second-order systems. Future work to either remedy some of these problems, or work that focuses on improving modal methods would be quite beneficial to the modeling and control problems considered in this work. 


\section{Bibliography}

[1] Peter Adolph, Willem Coppoolse, and Joseph Moerschell. Development and test of a fine pointing assembly for optical intersatellite link communications, 2002.

[2] P Benner, P Kürschner, and J Saak. Towards a hybrid balanced and modal truncation method for large-scale time invariant systems. Presentation Delivered at ModRed 2010 Workshop, 2010.

[3] D.F. Enns. Model reduction with balanced realizations: An error bound and a frequency weighted generalization. In Decision and Control, 1984. The 23rd IEEE Conference on, volume 23, pages 127-132, Dec 1984.

[4] Z.F. Fu and J. He. Modal Analysis. Elsevier Science, 2001.

[5] Seamus D Garvey, Michael I Friswell, and John E T. Penny. Clifford algebraic perspective on second-order linear systems. Journal of Guidance, Control, and Dynamics, 24(1):35-45, 2001.

[6] Keith Glover. All optimal hankel-norm approximations of linear multivariable systems and their l, -error bounds. International Journal of Control, 39(6):1115-1193, 1984.

[7] Y. Halevi. Approximated gramians and balanced realization of lightly 
damped flexible structures. Automatic Control, IEEE Transactions on, 47(1):193-198, Jan 2002.

[8] Joanna Iwaniec and Mickiewicz Alley. Novel approach to modal model reduction by means of the balanced realization method (part 1-theory). Molecular and Quantum Acoustics, 27:107-117, 2006.

[9] P. Koutsovasilis and M. Beitelschmidt. Comparison of model reduction techniques for large mechanical systems. Multibody System Dynamics, 20(2):111$128,2008$.

[10] Patrick Kürschner. Two-sided eigenvalue algorithms for modal approximation. PhD thesis, Masters thesis, Faculty of Math., Chemnitz Univ. of Technol., Chemnitz, 2010.

[11] Young W. Kwon and Hyochoong Bang. The Finite Element Method Using MATLAB. CRC Press, Inc., Boca Raton, FL, USA, 2nd edition, 2000.

[12] A.J. Laub, M.T. Heath, C. Paige, and R. Ward. Computation of system balancing transformations and other applications of simultaneous diagonalization algorithms. Automatic Control, IEEE Transactions on, 32(2):115-122, Feb 1987.

[13] C.T. Leondes. Electromechanical Systems: Mechatronic Systems, Techniques and Applications Volume Four. Gordon and Breach international series in engineering, technology, and applied science. Taylor \& Francis, 2000.

[14] Ching-An Lin and Tai-Yih Chiu. Model reduction via frequency weighted balanced realization. In American Control Conference, 1990, pages 20692070, May 1990. 
[15] MATLAB. version 7.10.0 (R2010a). The MathWorks Inc., Natick, Massachusetts, 2010.

[16] E.A. Mehiel. On Direct Model Reference Adaptive Controller Design for Flexible Space Structures. University of Colorado, 2003.

[17] D.G. Meyer and S. Srinivasan. Balancing and model reduction for secondorder form linear systems. Automatic Control, IEEE Transactions on, 41(11):1632-1644, Nov 1996.

[18] B. Moore. Principal component analysis in linear systems: Controllability, observability, and model reduction. Automatic Control, IEEE Transactions on, 26(1):17-32, Feb 1981.

[19] W.M.W. Muda, V. Sreeram, and H.H.-C. Iu. An improved algorithm for frequency weighted balanced truncation. In Decision and Control and European Control Conference (CDC-ECC), 2011 50th IEEE Conference on, pages 7182-7187, Dec 2011.

[20] Mayuresh J Patil. Decoupled second-order equations and modal analysis of a general nonconservative system. In Presented at the AIAA Dynamics Specialists Conference, 2000.

[21] L. Pernebo and L. Silverman. Model reduction via balanced state space representations. Automatic Control, IEEE Transactions on, 27(2):382-387, Apr 1982.

[22] Behnam Salimbahrami and Boris Lohmann. Order reduction of large scale second-order systems using krylov subspace methods. Linear Algebra and its Applications, 415(23):385 - 405, 2006. Special Issue on Order Reduction of Large-Scale Systems. 
[23] Q. Su, V. Balakrishnan, and C.-K. Koh. Efficient approximate balanced truncation of general large-scale rlc systems via krylov methods. In Design Automation Conference, 2002. Proceedings of ASP-DAC 2002. 7th Asia and South Pacific and the 15th International Conference on VLSI Design. Proceedings., pages 311-316, 2002.

[24] W. Wang and M.G. Safonov. Comparison between continuous and discretetime model truncation. In Decision and Control, 1990., Proceedings of the 29th IEEE Conference on, pages 494-499 vol.2, Dec 1990. 